\title{
On the continuum intensity distribution of the solar photosphere
}

\author{
S. Wedemeyer-Böhm ${ }^{1,2, \star}$ and L. Rouppe van der Voort ${ }^{1}$ \\ 1 Institute of Theoretical Astrophysics, University of Oslo, PO Box 1029 Blindern, 0315 Oslo, Norway \\ e-mail: sven . wedemeyer-bohm@astro.uio.no \\ 2 Center of Mathematics for Applications (CMA), University of Oslo, Box 1053 Blindern, 0316 Oslo, Norway
}

Received 2 March 2009 / Accepted 5 June 2009

\section{ABSTRACT}

\begin{abstract}
Context. For many years, there seemed to be significant differences between the continuum intensity distributions derived from observations and simulations of the solar photosphere.

Aims. In order to settle the discussion on these apparent discrepancies, we present a detailed comparison between simulations and seeing-free observations that takes into account the crucial influence of instrumental image degradation.

Methods. We use a set of images of quiet Sun granulation taken in the blue, green and red continuum bands of the Broadband Filter Imager of the Solar Optical Telescope (SOT) onboard Hinode. The images are deconvolved with point spread functions (PSF) that account for non-ideal contributions due to instrumental stray-light and imperfections. In addition, synthetic intensity images are degraded with the corresponding PSFs. The results are compared with respect to spatial power spectra, intensity histograms, and the centre-to-limb variation of the intensity contrast.

Results. The intensity distribution of SOT granulation images is broadest for the blue continuum at disc-centre and narrows towards the limb and for longer wavelengths. The distributions are relatively symmetric close to the limb but exhibit a growing asymmetry towards disc-centre. The intensity contrast, which is connected to the width of the distribution, is found to be $(12.8 \pm 0.5) \%$, $(8.3 \pm$ $0.4) \%$, and $(6.2 \pm 0.2) \%$ at disc-centre for blue, green, and red continuum, respectively. Removing the influence of the PSF unveils much broader intensity distributions with a secondary component that is otherwise only visible as an asymmetry between the darker and brighter than average part of the distribution. The contrast values increase to $(26.7 \pm 1.3) \%,(19.4 \pm 1.4) \%$, and (16.6 \pm 0.7$) \%$ for blue, green, and red continuum, respectively. The power spectral density of the images exhibits a pronounced peak at spatial scales characteristic for the granulation pattern and a steep decrease towards smaller scales. The observational findings like the absolute values and centre-to-limb variation of the intensity contrast, intensity histograms, and power spectral density are well matched with corresponding synthetic observables from three-dimensional radiation (magneto-)hydrodynamic simulations.

Conclusions. We conclude that the intensity contrast of the solar continuum intensity is higher than usually derived from groundbased observations and is well reproduced by modern radiation (magneto-)hydrodynamic models. Properly accounting for image degradation effects is of crucial importance for comparisons between observations and numerical models.
\end{abstract}

Key words. Sun: photosphere - radiative transfer

\section{Introduction}

Modern numerical simulations of convection in the upper solar atmosphere achieve a high degree of realism. For example, in their landmark paper, Stein \& Nordlund (2000) show that their MHD simulations are very successful at reproducing a number of observed properties of granulation. Other remarkable examples are the excellent agreement between observed and synthetic Fe line profiles (Asplund et al. 2000) and between observed and simulated magnetic faculae (Carlsson et al. 2004; Keller et al. 2004; Steiner 2005). As these diagnostic tests demonstrate the fidelity of the simulations, they can be used to achieve a deeper understanding of the physical mechanisms acting in the solar atmosphere and provide access to the un-observable subsurface regions. In recent years, extracting observables from simulations by synthesized spectra and intensity maps has grown into a powerful tool to understand and interpret observations (see, e.g., Khomenko et al. 2005; Langangen et al. 2007, and many more). Despite their success, there exist a number of diagnostic tests that show considerable discrepancy. One notorious example is the (rms) contrast of the continuum intensity emerging from the low solar photosphere, also referred to as granulation contrast or granular intensity fluctuation (see, e.g., Deubner \& Mattig 1975;

^ Marie Curie Intra-European Fellow of the European Commission.
Muller et al. 2007). Its value is straightforward to calculate and it is deeply connected to the basic mechanisms of convection as it is sensitive to the distribution of bright (hot) and dark (cool) surface structures. It is therefore troubling that contrast values derived from simulations do not agree with observed contrast values (see, e.g., Nordlund 1984; Keller 2006) with synthetic contrast values being usually significantly higher. For example, Uitenbroek et al. (2007) compared G-band images of the quiet Sun. They report contrasts of $14.1 \%$ for the (reconstructed) observed image and $21.5 \%$ for the synthetic image from a model by Stein \& Nordlund (2006).

Qualitatively, empirical studies show that the granulation contrast decreases with increasing wavelength and decreases with increasing distance from the disc-center. Wilken et al. (1997) used the German Vacuum Tower Telescope (VTT) to obtain granulation contrast at different heliocentric positions at a wavelength of $550 \mathrm{~nm}$. They derive $(13.5 \pm 1) \%$ for $\mu=1.0$ (disc-centre), $8-9 \%$ at $\mu=0.1$, and down to $3.8 \%$ very close to the solar limb at $\mu=0.07$. The wavelength dependence and center-to-limb variation of the contrast provide a stringent diagnostic test for simulations as it connects to the temperature stratification of the photosphere.

In observations many factors affect the contrast and most of them are poorly understood. Imperfections in the optics and straylight significantly reduce the contrast but are very difficult 

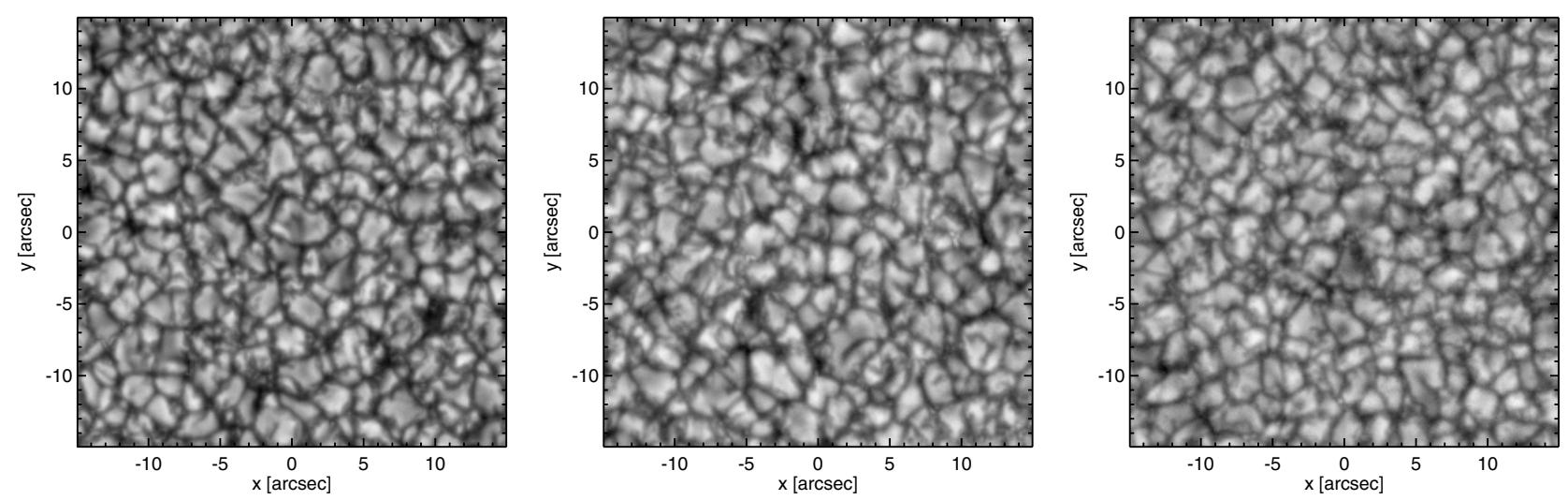

Fig. 1. SOT/BFI observations at disc-centre: Close-ups of intensity images in the blue (left), green (middle), and red (right) continuum band.

to measure and correct for. In addition, image degradation by the Earth atmosphere (seeing) has a huge impact on the image quality and can at best only partly be corrected for. The determination of the centre-to-limb variation of the mean absolute continuum intensity already requires enormous efforts (e.g., Neckel \& Labs 1984). Owing to these hardly controllable factors, a large range of contrast values are reported in the literature. See for example the review by Spruit et al. (1990) and more recently Table 2 by Sánchez Cuberes et al. (2000) and Fig. 1 by Kiselman (2008). Continuous advancement of observational methods, in particular the development of adaptive optics and image reconstruction techniques, lead to an increasing trend of the reported contrast values. These methods like for example speckle (e.g., von der Luehe 1993; de Boer 1993; Wöger et al. 2008) and Phase Diversity/MOMFBD (van Noort et al. 2005) involve measurements of the effective point-spread-function (PSF). Despite the high level of sophistication and impressive results, a complete reconstruction (i.e., full removal of the combined effect of seeing-induced degradation and instrumental aberrations) can never be obtained. Contributions to the PSF that are important for accurate photometry - essential for the determination of the granulation contrast - are often hardly known. This is in particular true for stray-light produced inside the optical instrument (Mattig 1983). Already a simple uniform intensity offset reduces the resulting intensity contrast. In most cases, however, the straylight contribution is likely to be anisotropic and varying over the field of view (FOV).

With the advent of the Solar Optical Telescope (SOT, Tsuneta et al. 2008; Ichimoto et al. 2008; Suematsu et al. 2008; Shimizu et al. 2008) onboard the Hinode spacecraft (Kosugi et al. 2007), the solar community has now access to space observations that have sufficient spatial resolution to resolve the scales that are important for an accurate determination of the granulation contrast. A space-born instrument has the obvious advantage of the absence of seeing so that only the instrumental PSF or stray-light needs to be considered. Wedemeyer-Böhm (2008, hereafter referred to as Paper I) derived a set of PSFs for the combined effect of the broadband filter imager (BFI) and the Optical Telescope Assembly (OTA) of SOT. He concluded that there is a small but significant amount of stray-light, although the instrument still operates close to the diffraction limit. The effect on the intensity distribution, including the granulation contrast, could thus be significant. This view is supported by Danilovic et al. (2008), who find that the difference in contrast between observations with the spectro-polarimeter (SP) of SOT and stateof-the-art numerical simulations is strongly reduced, when taking into account instrumental image degradation.
In this paper, we exploit the extensive Hinode data base that has been built up through its successful operations. Over time, the various observing programs have covered the quiet Sun at a whole range of observing angles providing the data to facilitate a robust statistical study of the wavelength dependence and centerto-limb variation of granulation contrast. Furthermore, we consider different independent numerical models. The paper is organised as follows: the observations are described in Sect. 2.1, followed by a description of the PSFs in Sect. 2.2 and the method of deconvolution in Sect. 2.3. The numerical simulations and the intensity synthesis are introduced in Sect. 3. The observations and the simulations are compared in detail for disc-centre images in Sect. 4, followed by an analysis of the centre-to-limb variation of the intensity distribution and the continuum intensity contrast in Sect. 5. Finally, the observations and simulations are compared in terms of spatial power spectral density in Sect. 6. Discussion and conclusions can be found in Sects. 7 and 8, respectively.

\section{Observations}

\subsection{SOT filtergrams}

The observations analysed in this study were obtained with the broadband filter instrument (BFI) of the solar optical telescope (SOT) onboard the Hinode satellite (see Sect. 1 for references) in the period from November 2006 until February 2008. Wideband filtergrams in the blue, green and red continuum from various dates are considered (see Fig. 1). The nominal central wavelengths of these filters are $450.45 \mathrm{~nm}, 555.05 \mathrm{~nm}$, and $668.40 \mathrm{~nm}$, respectively. The transmission profiles of these filters, which are available in Solar Soft (Freeland et al. 2000), have FWHMs of $0.22 \mathrm{~nm}, 0.27 \mathrm{~nm}$, and $0.31 \mathrm{~nm}$, respectively. As in Paper I, the position of the FOV and individual subregions on the solar disc is here reduced to the heliocentric position $\mu=\cos \theta$ with $\theta$ being the observing angle. It is justified by the finding that the intensity profiles across the solar limb do not show noticeable differences between different positions along the limb (N/W/S/E) (cf. Langhans \& Schmidt 2002). The FOV is $223^{\prime \prime} \times 112^{\prime \prime}$ for images that use the whole detector and $112^{\prime \prime} \times 112^{\prime \prime}$ for images that use only half. The pixel scale of the detector of only 0 ". 054 is so much smaller than the spatial resolution of the optical system $(0$ ". $2-0$ ". 3 , see, e.g., Shimizu et al. 2008 ) that images with $2 \times 2$ pixel-binning can be used next to unbinned images without causing significant differences in terms of contrast and intensity distribution. A total number of $584 \mathrm{im}$ ages of quiet Sun granulation are analysed, covering heliocentric 
positions from disc-centre to the solar limb. The images were selected to contain quiet Sun only, active region observations were excluded. Basic data processing (i.e., dark subtraction and flatfielding) was preformed using routines from the Hinode branch of Solar Soft.

\subsection{Point spread functions}

We use the point spread functions for the Solar Optical Telescope as they are calculated in Paper I. There are individual ideal and non-ideal PSFs for the blue, green, and the red continuum band. The ideal PSFs represent the image degradation due to diffraction at the main aperture stop of the telescope, which has a diameter of $50 \mathrm{~cm}$. The central obstruction and the spiders are taken into account. The non-ideal PSFs describe the combined effect of the Broadband Filter Imager and the Solar Optical Telescope. In contrast to the ideal PSFs, they account for contributions caused by imperfections and contamination of the optical components in the telescope (e.g., impurity of lens material, dust on optical surfaces, microscopic scratches or micro-roughness) and stray light, which may originate, e.g., from reflection at baffles. The non-ideal PSFs are calculated by convolution of the ideal diffraction-limited PSFs and Voigt profiles, which are chosen as model for the non-ideal contribution. For each wavelength channel, such theoretical point spread functions were produced for a grid of the parameters $\sigma$ and $\gamma$, which control the shape of the non-ideal Voigt-like contribution. These PSFs were then applied to artificial images of an eclipse and a Mercury transit. In a next step, the grid of resulting artificial intensity profiles across the terminators were compared to SOT observations of the Mercury transit from November 2006 and the solar eclipses from 2007. For each observed image in the blue, green, and red continuum channels of the BFI, the optimum PSF is indicated by the best-fitting artificial profile, resulting in the parameters $[\sigma, \gamma]$ and a usually small residual intensity offset. As the non-ideal contributions depend on a number of factors, the individual images produce a range of possible parameter combinations rather than a single solution. Nevertheless, the stray-light contributions are found to be best matched with Voigt functions with the parameters $\sigma=0$ ".008 and $\gamma=0$ ".004, 0 ".005, and 0 ".006 for the blue, green, and red continuum channels, respectively.

\subsection{Deconvolution}

The granulation images are deconvolved with (i) the ideal (diffraction-limited) PSFs and (ii) the non-ideal PSFs that are described in Sect. 2.2. We use all images at disc-centre for each wavelength channel (15 images each). The rectangular Hinode images were split into two square images for deconvolution in order to facilitate a straightforward execution and interpretation using standard FFT methods. After deconvolution, the average contrast of both sides is used for the analysis.

The deconvolution was performed by means of an optimal (Wiener) filter (see, e.g., Chapter 5.8 of Gonzalez \& Woods 2008). Figure 2 illustrates various aspects of the construction of the optimal filter. The graph shows the azimuthal average of the 2D power spectal density (or "power spectrum", see Sect. 6) of signal plus noise as the solid black line. In the signal-dominated regime, the power spectrum has a peak followed by decreasing power for the intermediate range of increasing wavenumbers $k$. In the noise-dominated regime, the power increases
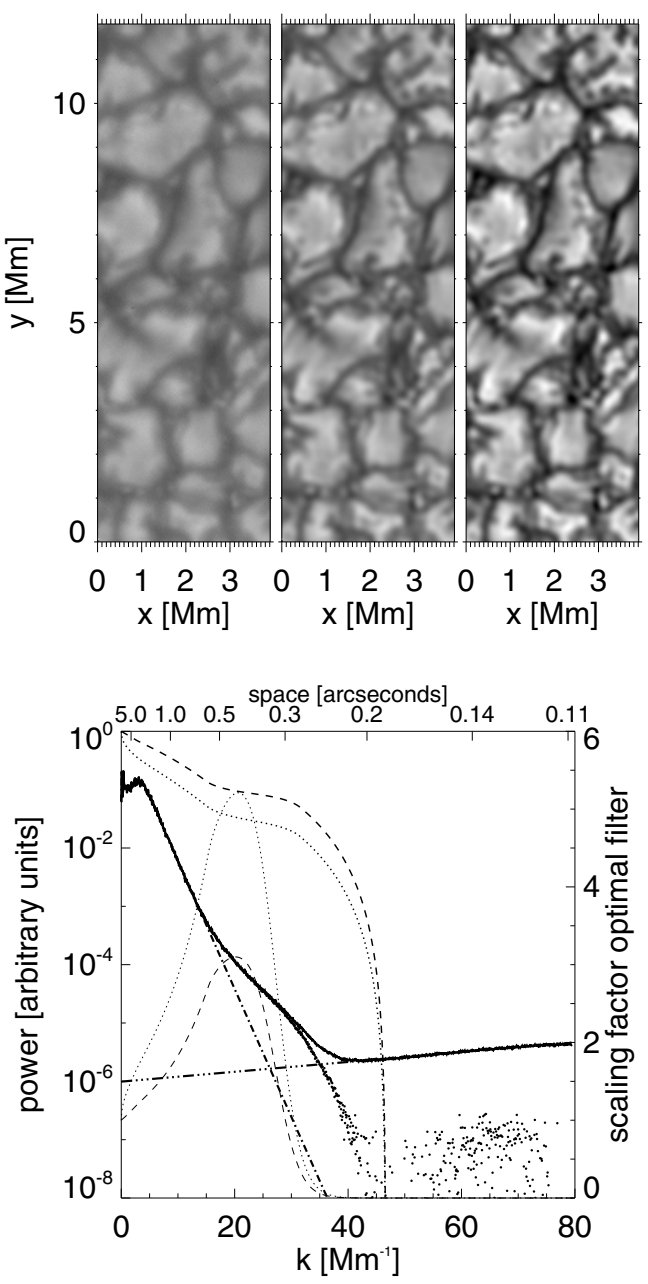

Fig. 2. Illustration of deconvolution using optimal (Wiener) filtering. The top panel shows a detail of a blue continuum disc center image. The left image is the flatfielded image, the middle image after deconvolution using the ideal PSF, and the right image after deconvolution using the non-ideal PSF from Paper I. All three images are scaled using the same intensity range: the minimum and maximum pixel values of the right panel. In the graph below, the thick solid line is the azimuthal average of the 2D power spectrum of a blue continuum disc center image. The triple-dot-dashed line is the noise model; the dots represent the power spectrum with the noise model subtracted. The dash-dot line is the model for the signal in the absence of noise. Lines in medium thickness: the dashed line is the ideal MTF, the dotted line the non-ideal MTF. Both have their cut-off at the diffraction limit $\lambda / D=0$ ". 19 . Thin lines: the dashed line is the optimal filter for the ideal PSF, the dotted line for the non-ideal PSF. Both lines relative to the $y$-axis scaling on the right side.

with increasing wavenumber for spatial scales smaller than the diffraction limit $\lambda / D<0$ ". 19 (see the cut-off for the MTF).

For the construction of the optimal filter, an assumption has to be made for the power spectrum of the signal in the absence of noise (see the discussion in Chapter 13.4 of Press et al. 1992). To this end, a noise model was constructed by extrapolating a fit to the noise tail into the signal region (triple-dot-dashed line). The subtraction of the noise model from the measured power spectrum is shown as black dots. The power spectrum displays an excess in the form of a less steep power fall-off around spatial scale 0 ". 3 . A similar excess is seen in all power spectra and becomes more pronounced for the green and red continuum channels. Since we do not observe such excess in power spectra from higher spatial resolution granulation images from the Swedish 
1-m Solar Telescope (see, e.g., Rouppe van der Voort et al. 2004) and the Dunn Solar Telescope (see, e.g., Wöger et al. 2008), we decided to exclude this excess from the signal model. The origin of the excess is unclear but we can exclude JPEG compression artifacts (see Sect. 7.3). The signal model is derived from an extrapolation of the power spectrum to high wave numbers following the power law in the $\left[00^{\prime \prime} 6-1^{\prime \prime}\right]$ range (dash-dotted line).

The azimuthal average of the optimal filter is also shown in the graph of Fig. 2 (thin lines, following the right $y$-axis scaling). The filter enhances $(>1)$ for spatial scales $\geq 0$ ". 27 and suppresses noise for smaller scales. Although the shape of the optimal filter is significantly affected by in- or exclusion of the power excess in the signal model (including the excess shifts enhancement of the filter down to $0{ }^{\prime \prime} .2$ ), the contrast is affected only little: $0.1 \%$ higher contrast ${ }^{1}$ for the ideal PSF and $0.2 \%$ for the non-ideal PSF. This illustrates that the contribution of small spatial scales to the overall contrast is limited. That makes deconvolution by an optimal filter a robust procedure, where details of the construction of the filter have only limited impact on the contrast values.

While the deconvolution of disc-centre images produces rather robust results, the treatment of images at smaller $\mu$ is problematic. In particular, images close to the solar limb cover a significant range in $\mu$. Consequently, the properties of the stray light component vary significantly across the FOV. The same applies to the noise in power spectra that are used for the construction for the Wiener filter. It is therefore not possible to construct a Wiener filter that is optimal for the whole FOV. Retreating to deconvolution of smaller image sections with small intrinsic variation in $\mu$ would cause systematic errors due to neglect of the far PSF wings. The contrast values for deconvolved images away from the disc-centre would suffer a pronounced spread and possibly little significance only. We therefore restrict the deconvolution to disc-centre images.

The three images in Fig. 2 show the effect of the deconvolution. The left panel shows the flatfielded image, the middle panel after deconvolution considering the ideal PSF, and the right panel after considering the non-ideal PSF from Paper I. All images are shown using the same intensity scaling. The effect of noise suppression for the two right panels is clear. The contrast is enhanced from $12.6 \%$ for the flatfielded image to $17.9 \%$ for the image deconvolved with the ideal PSF, and to $26.5 \%$ after deconvolution with the non-ideal PSF. See Table 3 for statistics for all disc-centre images.

\section{Synthetic intensity}

\subsection{Numerical simulations}

An overview over the models used here is given in Table 1. Most of our analysis will be based on three snapshots of a recent 3D radiation hydrodynamic simulation by Steffen (2007), which was computed with $\mathrm{CO}^{5} \mathrm{BOLD}$ (Freytag et al. 2002; Wedemeyer et al. 2004; Freytag et al. 2008). The model, which is identified as $\mathrm{Ch} 0$ hereafter, has a horizontal grid spacing of $28 \mathrm{~km}$ and an extent of $11.2 \mathrm{Mm} \times 11.2 \mathrm{Mm}$. It reaches from $\sim-2400 \mathrm{~km}$ below the average height of optical depth $\tau_{500 \mathrm{~nm}}=1$ to $+750 \mathrm{~km}$ above. The simulation code $\mathrm{CO}^{5}$ BOLD employs a Riemanntype solver. Note that the effective spatial resolution of models produced with such a code is higher than for models calculated with a finite-differences solver at same grid spacing. The

\footnotetext{
1 All contrast values and differences between them are given in percent points, notified by $\%$ (see Sect. 5.2).
}

Table 1. Numerical simulations used in this study.

\begin{tabular}{|c|c|c|c|c|}
\hline \multirow[b]{2}{*}{ ID } & \multirow[b]{2}{*}{ Reference } & \multicolumn{3}{|c|}{ Parameters } \\
\hline & & $\mathrm{RT}^{a}$ & $\begin{array}{c}\left\langle B_{z}\right\rangle^{b} \\
{[\mathrm{G}]}\end{array}$ & $\begin{array}{c}\text { hor. ext. }{ }^{c} \\
{[\mathrm{Mm}]}\end{array}$ \\
\hline \multicolumn{5}{|c|}{$\mathrm{CO}^{5} \mathrm{BOLD}$ simulations } \\
\hline $\mathrm{Ch} 0$ & Steffen (2007) & non-gr. & 0 & $11.2 \times 11.2$ \\
\hline Ch1 & Wedemeyer et al. (2004) & grey & 0 & $5.6 \times 5.6$ \\
\hline $\mathrm{Ch} 2$ & Wedemeyer et al. (2004) & non-gr. & 0 & $5.6 \times 5.6$ \\
\hline $\mathrm{Cm}$ & Schaffenberger et al. (2006) & grey & 10 & $4.8 \times 4.8$ \\
\hline \multicolumn{5}{|c|}{ Stein \& Nordlund simulations } \\
\hline $\mathrm{SNh}$ & Stein \& Nordlund (2006) & non-gr. & 0 & $6.3 \times 6.3$ \\
\hline $\mathrm{SNm}$ & Stein \& Nordlund (2006) & non-gr. & 250 & $6.3 \times 6.3$ \\
\hline
\end{tabular}

${ }^{a}$ For each model it is indicated if frequency-independent ("grey") or -dependent ("non-grey") radiative transfer (RT) was considered.

${ }^{b}$ Magnetic field $\left\langle B_{z}\right\rangle$.

${ }^{c}$ Horizontal extent of the computational domain.

radiative transfer in the simulation uses a frequency-dependent ("non-grey") long characteristics scheme with multi-group opacities with five bins. The latter are constructed from updated MARCS opacities (see Gustafsson et al. 2008, and references therein).

For comparison, additional sets of synthetic images are calculated for $\mathrm{CO}^{5} \mathrm{BOLD}$ models and simulations by Stein \& Nordlund (1998). All sets consist of three snapshots each. The average contrast and standard variation for disc-centre images are given in Table 3. The abbreviations $\mathrm{Ch} 1$ and $\mathrm{Ch} 2$ stand for the non-magnetic models by Wedemeyer et al. (2004) with frequency-independent ("grey") and frequency-dependent ("non-grey") radiative transfer, respectively. $\mathrm{Cm}$ is also a $\mathrm{CO}^{5}$ BOLD model but with a weak magnetic field of $\left\langle B_{z}\right\rangle=10 \mathrm{G}$ (Schaffenberger et al. 2006). The models by Stein \& Nordlund only differ such that $\mathrm{SNh}$ has no magnetic field, whereas $\mathrm{SNm}$ has $\left\langle B_{z}\right\rangle=250 \mathrm{G}$. A snapshot of the latter has already been used by, e.g., Carlsson et al. (2004), Leenaarts et al. (2006), and by Uitenbroek et al. (2007). See also Stein \& Nordlund (2006).

\subsection{Intensity synthesis}

We use the spectrum synthesis code Linfor3D to produce synthetic intensity maps for the radiation (magneto-)hydrodynamic simulations listed in Table 1. Linfor3D (see http://www . aip. $\mathrm{de} / \sim \mathrm{mst} /$ infor3D_main.html) is originally based on the Kiel code LINFOR/LINLTE. It solves the detailed radiative transfer in full 3D for an input atmosphere under the assumption of local thermodynamic equilibrium (LTE). The computations are performed on a numerical grid that has a higher resolution compared to the input model. For consistency reasons, the same opacities and element abundances are used in the numerical simulations and in Linfor3D (e.g., updated MARCS opacities for model Ch0, see Sect. 3.1).

The green and in particular the red continuum band contain only a few mostly weak spectral lines so that their relative influence on the radiation integrated over the whole wavelength band is negligible. The blue continuum channel includes a spectral line of neutral iron (FeI) at $450.483 \mathrm{~nm}$, which we explicitly take into account. The line parameters are taken from the Vienna Atomic Line Database (Kupka et al. 1999). The influence of the line on the radiation integrated over the full blue continuum filter turns out to be negligible. The change in contrast would be well 

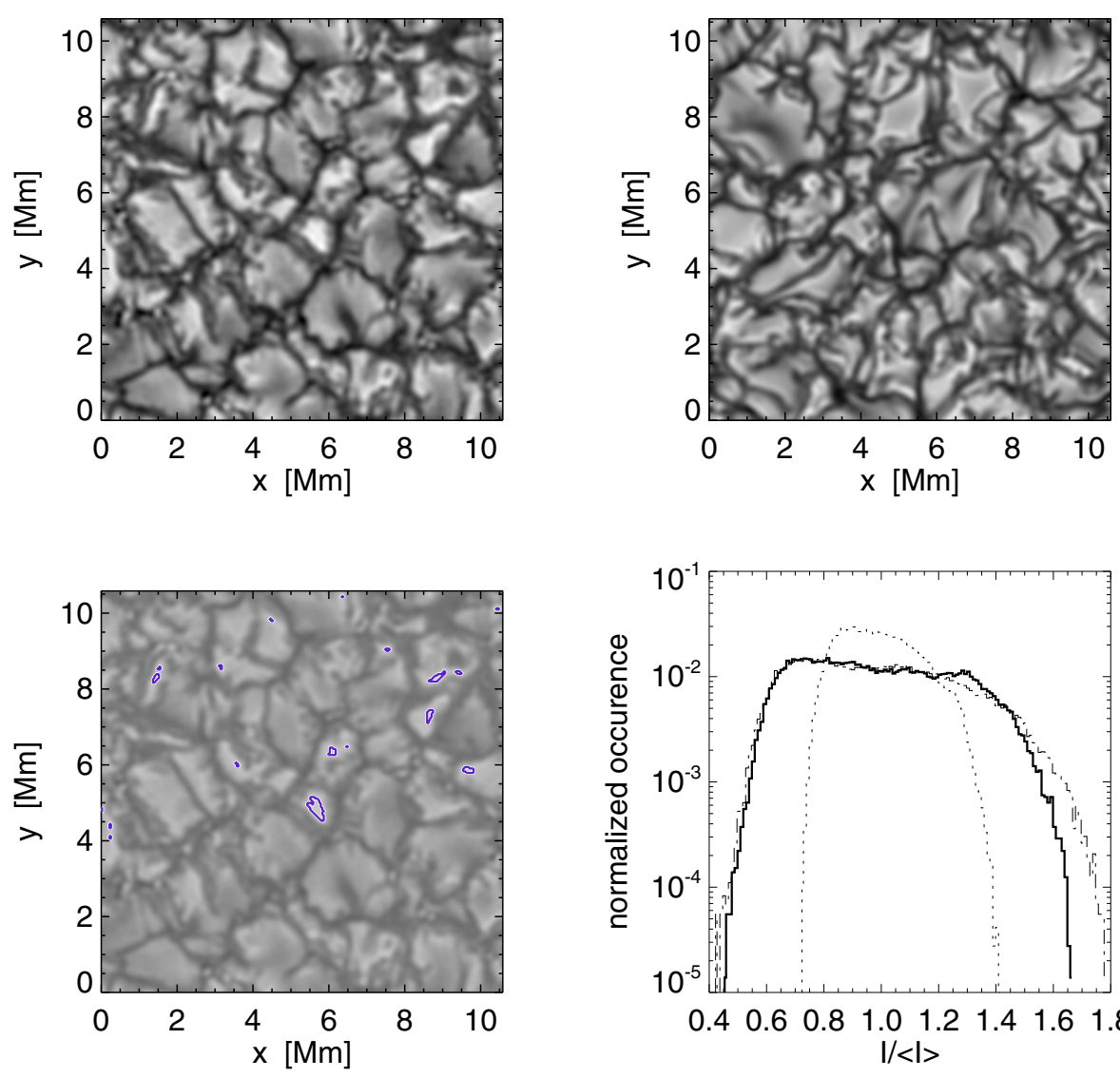

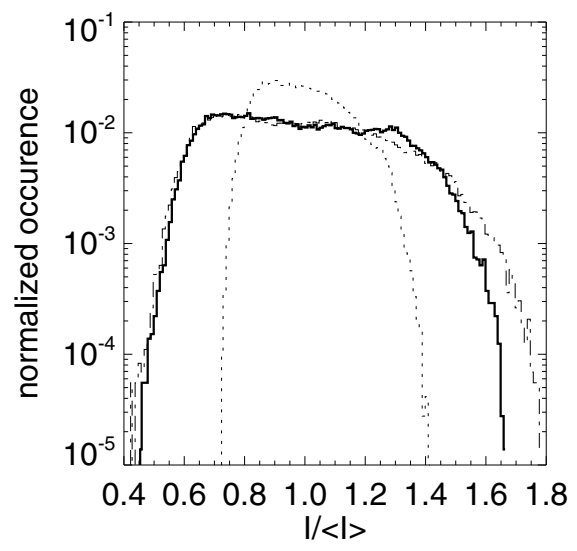

Fig. 3. Comparison of synthetic and observed images for the blue continuum channel. Top left: observed deconvolved image, top right: synthetic image rescaled to the same pixelsize as the observed image; bottom left: same as top left but with contours enclosing the pixels, which are brighter than in the synthetic image $(I /\langle I\rangle>1.66)$. The image is made fainter for better visibility of the contours. Bottom right: histograms for the intensity distributions: original observed (dotted), deconvolved (dotdashed), and original synthetic intensity (thick solid). below 0.1 percent point with respect to pure continuum images and also the intensity histograms are barely affected.

\subsection{Degradation of synthetic maps}

The PSFs introduced in Sect. 2.2 are used to simulate the image degradation caused by the SOT/BFI optics. Each synthetic intensity map is convolved with the non-ideal PSF of the corresponding wavelength. For the model $\mathrm{Ch} 0$, we also use the ideal PSFs and two different FOVs (see discussion in Sect. 7.3).

\section{Comparison of observed and synthetic images}

We begin our comparison of the Hinode filtergrams and the numerical simulations on the basis of disc-centre images. First, the de-convolved observations are opposed to the original synthetic intensity maps from model $\mathrm{Ch} 0$ in Sect. 4.1. The original observations are then checked against the corresponding degraded synthetic maps in Sect. 4.2.

\subsection{Synthetic images compared to deconvolved observations}

The synthetic images have a lower contrast than the PSF deconvoluted SOT/BFI images but are still within the 1- $\sigma$ variation of the observed values in the blue and green continuum. The differences are $1.7 \%, 1.3 \%$, and $1.9 \%$ for the three channels, respectively. The comparison is illustrated in Fig. 3 for blue continuum images at disc-centre.

In order to make a fair comparison, the synthetic image is filtered with a low-pass filter. The filtering accounts for the fact that the observations are taken with a telescope with finite aperture.
Consequently, there can be no higher spatial frequencies present than given by the size of the aperture. In addition, the observations contain noise at a level that affects the highest spatial frequencies (the power spectrum of the image is dominated by noise at frequencies higher than the diffraction limit). The lowpass filter was constructed in a way such that the power spectrum of the filtered synthetic image matches the power spectrum of the deconvolved image. After convolution, the synthetic image was re-sampled to the Hinode pixel scale. The observed image was selected to be as quiet as possible, selecting a region with as few magnetic bright points as possible. The top left image in Fig. 3 shows the deconvolved observed image, the top right image the filtered synthetic image. Both are shown using the same intensity scaling. In a visual comparison of the two images, one can distinguish the observed from the synthetic image by the presence of a noise pattern that remains after application of the optimal filter. Other than this noise pattern, the two images are qualitatively remarkably similar.

The lower right panel of Fig. 3 shows the histograms of the intensity values of the two images. In addition, the histogram of the flatfielded observed image is shown (dashed line). Deconvolution by the non-ideal PSF has a pronounced effect on the intensity histogram. The histograms of the deconvolved observed and synthetic images are very similar. It should be noted that the low-pass filtering of the synthetic image has only limited effect on the histogram. Both the synthetic images and the deconvolved observations exhibit an essentially "double-peaked" distribution, although the peak at $I /\langle I\rangle<1$ is much more pronounced than the secondary peak at $I /\langle I\rangle>1$. The latter can easily be obscured and only be noticable as asymmetry in the distribution (see Fig. 6). The distribution can actually be decomposed in a bright granular and a dark intergranular component for the simulations, when one considers the sign of the flow 

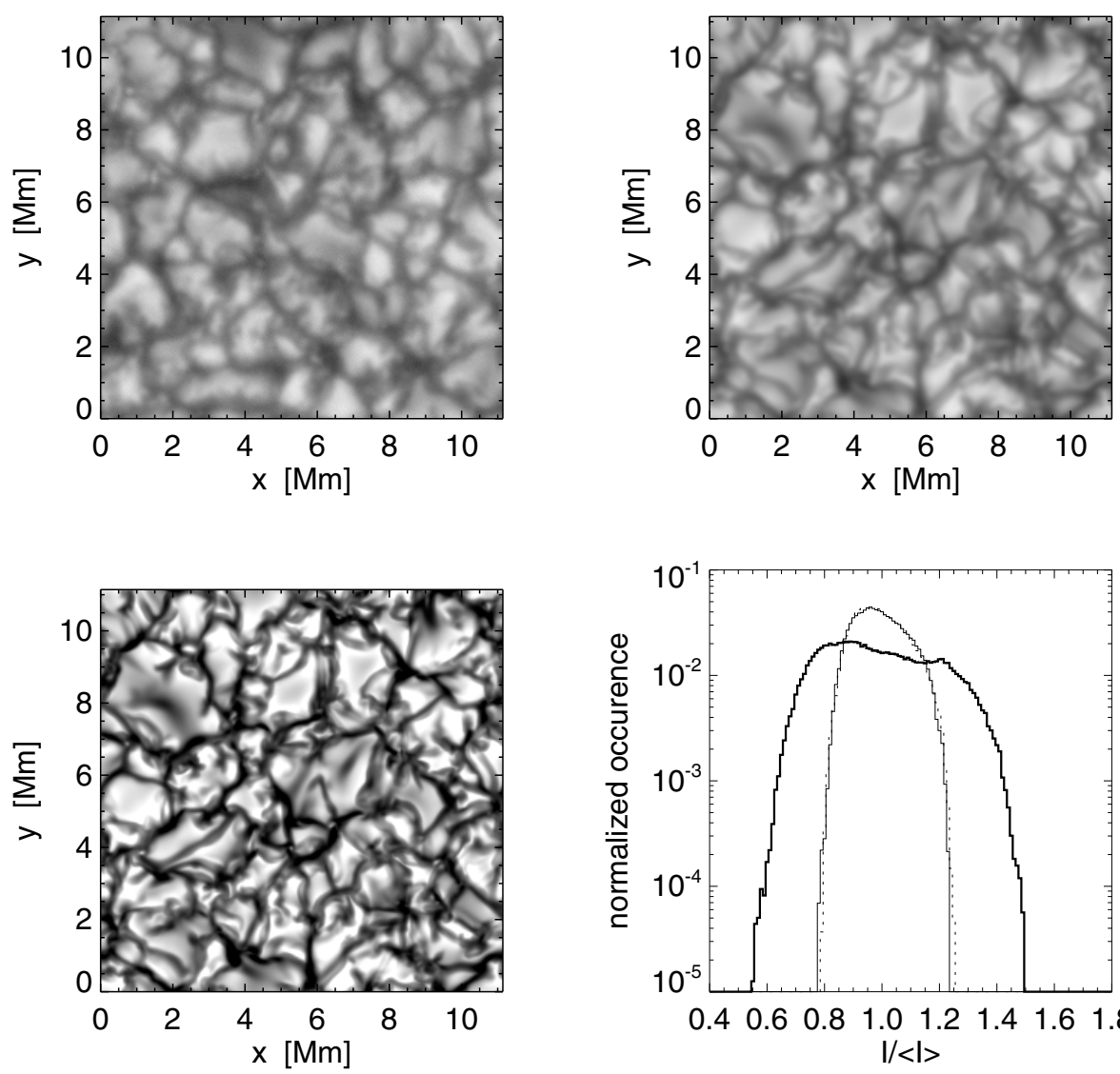

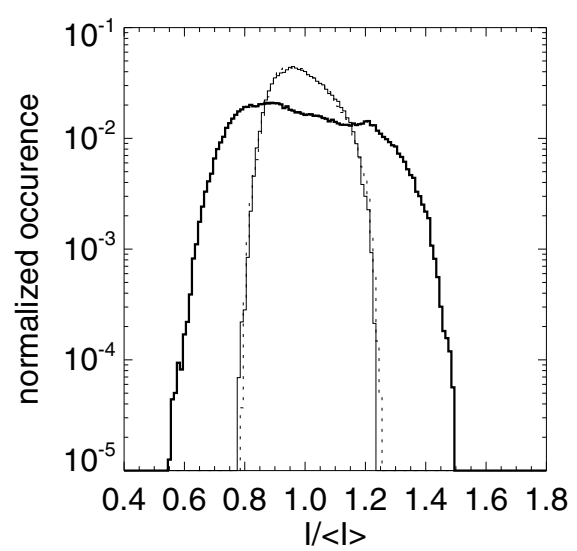

Fig. 4. Comparison of synthetic and observed images for the green continuum channel. Top left: original observed image, top right: degraded synthetic image rescaled to the same pixelsize as the observed image; bottom left: original synthetic image; bottom right: histograms for the observed intensity (dotted), degraded synthetic intensity (thin solid), and original synthetic intensity (thick solid). velocity (downflow/upflow). This property has been known for some time (see, e.g., Fig. 3 in Stein \& Nordlund 2000). A remarkable difference between the two histograms, however, can be seen at high intensity values. These high pixel values (160 in total, or $0.2 \%$ ) can be found as bright regions inside granules, enclosed by the contours in the lower left panel of Fig. 3.

\subsection{Degraded images compared to original observations}

Now we reverse the procedure by artificially degrading the synthetic images by convolution with the non-ideal PSFs described in Paper I. Using the best-fit PSFs with Voigt contributions, reduces the contrast values of the synthetic image to $13.0 \%, 8.6 \%$, and $6.0 \%$ (average over three snapshots) for the blue, green, and red continuum, respectively (see Table 3 ). The resulting differences between the average observational and degraded synthetic values are then $0.2,0.3$, and -0.2 percent points for blue, green, and red continuum, respectively. The above mentioned degradation was performed with a PSF extending with a size given by the computational box of the model, i.e., $15^{\prime \prime} .4 \times 15^{\prime \prime} 4$. The far wings of the PSF are this way neglected. We therefore repeat the degradation with a PSF with a size of $110^{\prime \prime} \times 110^{\prime \prime}$, which is comparable to the FOV of the observations. The synthetic image is repeated periodically to fill the FOV. This procedure is warranted as periodic boundaries were used during the simulation run. The large synthetic images are now degraded with the full non-ideal PSF, including the far wings. At disc-centre, we find mean contrast values of $11.3 \%, 7.4 \%$, and $5.1 \%$ for the blue, green, and red continuum, respectively (see Table 3 ). This corresponds to an effective reduction of the contrast ranging from 0.87 to 0.85 of the value for the small FOV. On the other hand, uncertainties in the parameters for the model of the non-ideal PSF contributions have a tendency to increase the contrast again towards the value found for the small FOV (see Sect. 7.3). We conclude that the observed and synthetic snapshots agree well in terms of intensity contrast when considering the uncertainties inherent to the applied PSF.

Figure 4 shows examples for an original observed and a synthetic image in the green continuum channel. The intensity distributions of the original observed and the degraded synthetic image agree well. The distributions of individual images and subfields, however, vary to some degree in accordance to the variation in intensity contrast. Some cases show a narrower distribution, some exhibit a number of particularly bright pixels (as in Fig. 3 for the deconvolved image), while the secondary component of brighter than average pixels (see Sect. 4.1) is sometimes only noticeable as a distribution asymmetry. The distributions are generally close to those found for the synthetic images. See Fig. 5 for a comparison of simulation and observation that includes all available disc-centre images.

\section{Centre-to-limb variation of the continuum intensity distribution}

In this section, the properties of the observed and simulated intensity distribution and contrast are investigated as function of heliocentric position.

\subsection{Observed intensity histograms}

The intensity distribution of the original (reduced) SOT images is illustrated in Fig. 6 for the three continuum wavelengths. The pixels of all images are sorted by disc position $\mu$ into bins of size $\Delta \mu=0.05$. The upper row of the figure shows the histograms for all bins, whereas the rows below show histograms for selected 

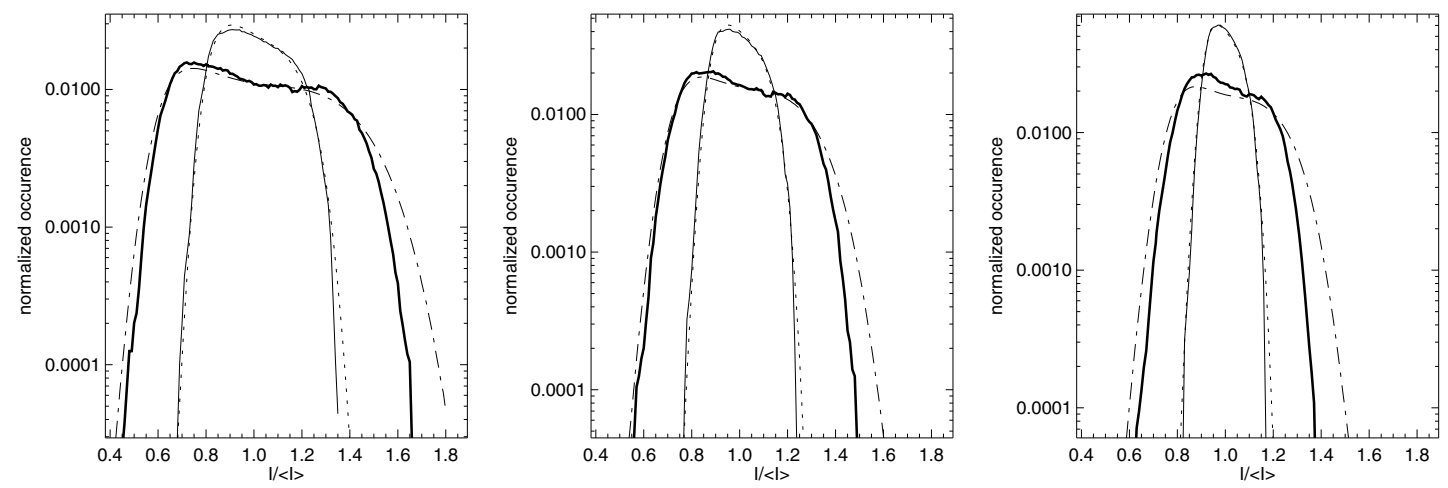

Fig. 5. Comparison of the intensity distribution in all synthetic and observed disc-centre images for the blue (left), green (middle), and red continuum channel (right). Shown are the distributions for the original observed images (dotted), the deconvolved images (dot-dashed), the degraded synthetic images (thin solid), and the original synthetic intensity maps (thick solid).
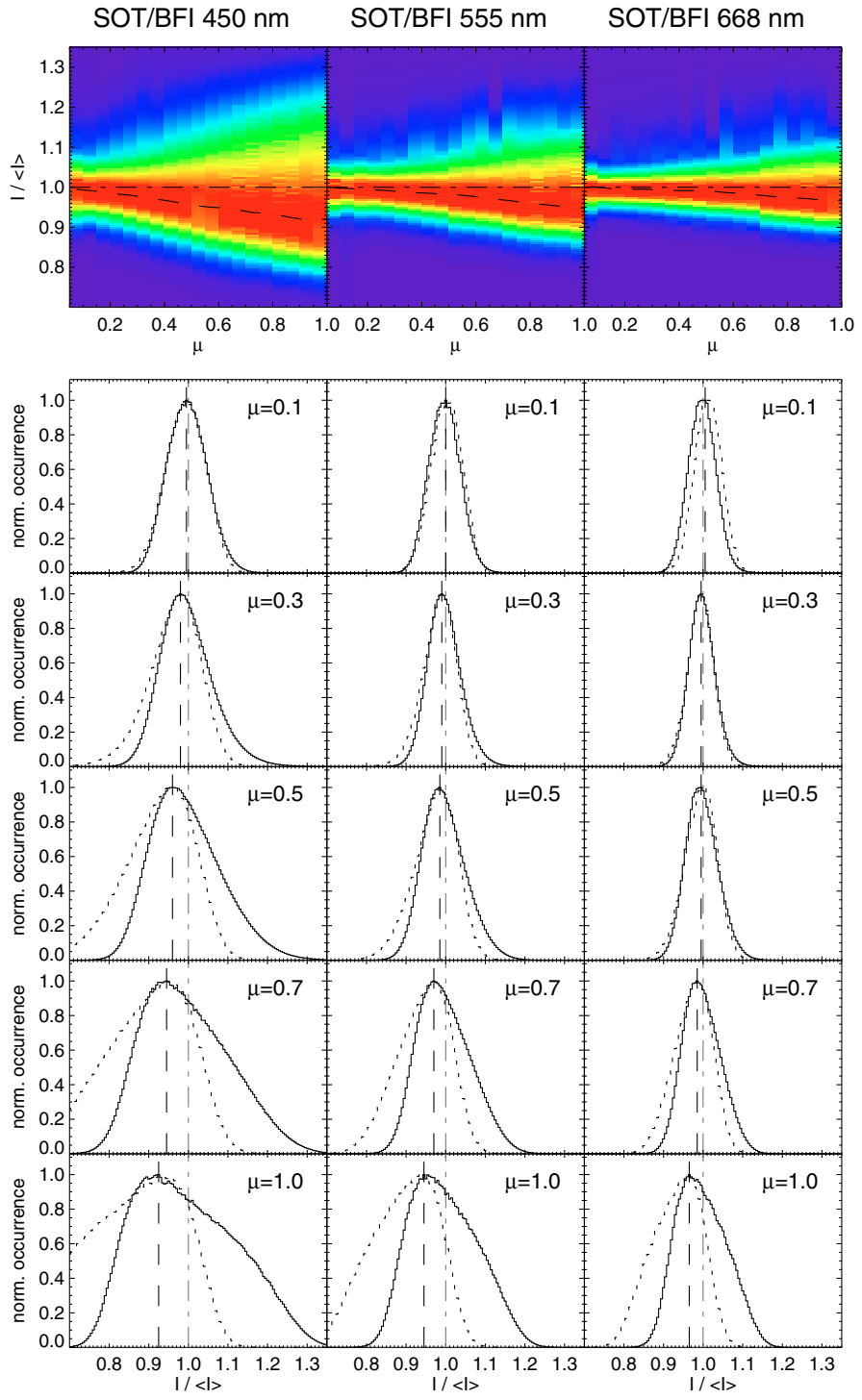

Fig. 6. Intensity histograms for the blue (left column), green (middle column), and red continuum (right column) as function of disc-position $\mu$. Top row: histograms for all $\mu$ with the relative occurrence as color/greyshades. Rows below: histograms for $\mu=0.1,0.3,0.5,0.7$, and 1.0, respectively. The lines mark $I /\langle I\rangle=1$ (dot-dashed), the histogram peaks (dashed), and - in the lower rows - the histograms mirrored at the histogram peaks (dotted). The latter reveals the asymmetry of the distribution. heliocentric positions. The intensity distribution becomes narrower with increasing wavelength and with decreasing $\mu$. The histograms are relatively symmetric close to the limb but exhibit a growing asymmetry towards disc-centre. The asymmetry is most pronounced at $\mu=1.0$. Generally, there seems to be an increasing number of pixels with intensities brighter than average, which cause the distribution to be broader at disc-centre. Consequently, the peaks of the histograms move from around $I /\langle I\rangle \approx 1.0$ at the limb to smaller values at disc-centre.

Removing the PSF (see Sect. 2.3) from the images has a dramatic effect on the resulting intensity distribution. It broadens, covering a much larger range in $I /\langle I\rangle$, and exhibits a subtle secondary peak for brighter than average pixels. More details are discussed in comparison to synthetic images in Sect. 4 and Fig. 3.

\subsection{Observed intensity contrast}

Definition. The intensity contrast is defined as

$\delta I_{\mathrm{rms}} \equiv \frac{\sqrt{\frac{1}{N} \sum_{x, y}\left(I(x, y)-\langle I\rangle_{x, y}\right)^{2}}}{\langle I\rangle_{x, y}}$

where $I(x, y)$ are the intensity values of the individual pixels with the average $\langle I\rangle_{x, y}$ over all $N$ pixels.

Contrast of reduced images. The contrast values are listed for the three considered BFI continuum bands and different heliocentric positions $\mu$ in Table 2 . The average contrast values for the SOT filtergrams are derived from regions of equal $\mu$ with a bin size of $\Delta \mu=0.05$ for all images. At disc-centre, the images are within the same $\mu$-bin, whereas image segments are sorted into the bins closer to the limb. The complete images at disc-centre (15 for each wavelength), produce the following average contrasts and standard deviations: $(12.8 \pm 0.5) \%,(8.3 \pm 0.4) \%$, and $(6.2 \pm 0.2) \%$ for blue, green, and red continuum, respectively. The intensity contrast strongly decreases towards the longer wavelengths. The same behavior is found for all $\mu$. For each wavelength, the contrast also decreases from centre to limb, i.e. with decreasing $\mu$, which can also be seen from the width of the histograms discussed in Sect. 5.1. This centre-to-limb variation of the contrast is shown in Fig. 7, which can be interpreted as grey-shaded histograms. The histograms for the individual bins exhibit a maximum close to the corresponding average contrast (solid circles, cf. Table 2). The distribution, however, cannot be 
Table 2. Intensity contrast $\delta I_{\mathrm{rms}}$ in percent points for the original observations in the three considered BFI continuum bands.

\begin{tabular}{ccccccc}
\hline \hline $\begin{array}{c}\delta I_{\mathrm{rms}}^{a} \\
\mu^{b}\end{array}$ & $450.45 \mathrm{~nm}$ & $555.00 \mathrm{~nm}$ & $668.40 \mathrm{~nm}$ \\
\hline $0.05-0.10$ & 4.8 & $(5.0)$ & 3.7 & $(3.8)$ & 3.2 & $(3.2)$ \\
$0.10-0.15$ & 5.1 & $(5.1)$ & 3.7 & $(3.6)$ & 2.9 & $(3.2)$ \\
$0.15-0.20$ & 5.5 & $(5.4)$ & 4.0 & $(4.0)$ & 3.1 & $(3.2)$ \\
$0.20-0.25$ & 6.1 & $(6.0)$ & 4.0 & $(4.0)$ & 3.2 & $(3.2)$ \\
$0.25-0.30$ & 6.7 & $(6.6)$ & 4.3 & $(4.1)$ & 3.4 & $(3.2)$ \\
$0.30-0.35$ & 7.3 & $(7.4)$ & 4.6 & $(4.6)$ & 3.5 & $(3.6)$ \\
$0.35-0.40$ & 7.9 & $(8.0)$ & 4.9 & $(4.8)$ & 3.8 & $(3.5)$ \\
$0.40-0.45$ & 8.4 & $(8.2)$ & 5.2 & $(5.1)$ & 4.0 & $(3.8)$ \\
$0.45-0.50$ & 8.9 & $(8.9)$ & 5.4 & $(5.4)$ & 4.1 & $(4.0)$ \\
$0.50-0.55$ & 9.5 & $(9.7)$ & 6.1 & $(6.1)$ & 4.2 & $(4.4)$ \\
$0.55-0.60$ & 10.0 & $(10.4)$ & 6.2 & $(6.4)$ & 4.3 & $(4.3)$ \\
$0.60-0.65$ & 10.4 & $(9.9)$ & 6.4 & $(6.6)$ & 4.5 & $(4.5)$ \\
$0.65-0.70$ & 10.8 & $(10.7)$ & 6.6 & $(6.4)$ & 4.7 & $(4.7)$ \\
$0.70-0.75$ & 11.1 & $(11.0)$ & 7.1 & $(7.1)$ & 5.3 & $(5.4)$ \\
$0.75-0.80$ & 11.3 & $(11.4)$ & 7.5 & $(7.5)$ & 5.5 & $(5.6)$ \\
$0.80-0.85$ & 11.9 & $(11.6)$ & 7.7 & $(7.4)$ & 5.7 & $(5.6)$ \\
$0.85-0.90$ & 12.1 & $(12.2)$ & 7.9 & $(7.8)$ & 5.9 & $(5.8)$ \\
$0.90-0.95$ & 12.4 & $(12.5)$ & 7.9 & $(7.8)$ & 6.1 & $(6.2)$ \\
$0.95-1.00$ & 12.8 & $(13.1)$ & 8.3 & $(8.4)$ & 6.2 & $(6.2)$ \\
\hline
\end{tabular}

${ }^{a}$ The average contrast in each bin is given next to the peak of the corresponding contrast histograms (in parentheses, compare to Fig. 7).

${ }^{b}$ Heliocentric positions $\mu$; The $\mu$ bins $(\Delta \mu=0.05)$ are the same as in Fig. 7.

described by means of a, e.g., simple Gaussian. The contrast distribution for the blue continuum even shows a small number of cases with enhanced contrast, which manifest as a secondary ridge in Fig. 7a. This is most likely connected to the influence of magnetic fields, e.g., in the form of network bright points, which are unavoidable for the large size of the used FOV, even though enhanced network regions were explicitly excluded from the sample (see Sect. 4). Small errors in the determination of the limb and also the limited spatial resolution of SOT introduce uncertainties for the determination of the contrast very close to the limb (see Sect. 7.3). Data for $\mu<0.07$ should thus be interpreted with caution.

Contrast after PSF deconvolution. Removing the instrumental influence by deconvolution with the PSF has a dramatic effect on the contrast. The results are summarised in Table 3. The contrast of blue continuum images at disc-centre is increased by 5.2 percent points after deconvolution with the diffractionlimited ideal PSF and another 8.7 points, when the full non-ideal PSF is used. In total, the original contrast is more than doubled to $(26.7 \pm 1.3) \%$. The green and red channel show similarly large effects. The contrast increases from $(8.3 \pm 0.4) \%$ to $(19.4 \pm 1.4) \%$ in the green and from $(6.2 \pm 0.2) \%$ to $16.6 \pm 0.7) \%$ in the red channel.

Error estimate. It is not trivial to give a reliable error estimate for the empirical contrast values. Next to systematic errors due to instrumental properties (PSF, straylight, see Sect. 7.3), there are statistical errors arising from the selection of the pixel ensembles. The contrast values given in Table 2 are derived from
Table 3. Average contrast $\delta I_{\text {rms }}$ and standard deviation of the original and the deconvolved disc-centre observations in comparison with the synthetic images.

\begin{tabular}{|c|c|c|c|c|c|c|}
\hline \multirow{2}{*}{$\begin{array}{l}\delta I_{\mathrm{rms}} \\
{[\%]}\end{array}$} & \multicolumn{6}{|c|}{$\lambda$} \\
\hline & \multicolumn{2}{|c|}{$450.45 \mathrm{~nm}$} & \multicolumn{2}{|c|}{$555.00 \mathrm{~nm}$} & \multicolumn{2}{|c|}{$668.40 \mathrm{~nm}$} \\
\hline \multicolumn{7}{|c|}{ observation } \\
\hline original & 12.8 & \pm 0.5 & 8.3 & \pm 0.4 & 6.2 & \pm 0.2 \\
\hline ideal PSF & 18.0 & \pm 0.8 & 12.3 & \pm 0.8 & 9.9 & \pm 0.4 \\
\hline full PSF & 26.7 & \pm 1.3 & 19.4 & \pm 1.4 & 16.6 & \pm 0.7 \\
\hline \multicolumn{7}{|c|}{ simulation Ch0 (Steffen 2007) ${ }^{a}$} \\
\hline original & 25.0 & \pm 0.1 & 18.1 & \pm 0.1 & 13.8 & \pm 0.1 \\
\hline ideal PSF & 16.7 & \pm 0.3 & 11.4 & \pm 0.2 & 8.2 & \pm 0.2 \\
\hline non-ideal PSF (1) & 13.0 & \pm 0.3 & 8.6 & \pm 0.2 & 6.0 & \pm 0.2 \\
\hline non-ideal PSF (2) & 11.3 & \pm 0.2 & 7.4 & \pm 0.2 & 5.1 & \pm 0.2 \\
\hline \multicolumn{7}{|c|}{${\text { other simulations (original })^{b}}^{b}$} \\
\hline Ch1 & 25.8 & \pm 0.5 & 19.0 & \pm 0.5 & 14.6 & \pm 0.5 \\
\hline $\mathrm{Ch} 2$ & 25.5 & \pm 0.5 & 18.4 & \pm 0.5 & 13.9 & \pm 0.5 \\
\hline $\mathrm{Cm}$ & 25.4 & \pm 0.1 & 19.0 & \pm 0.5 & 14.7 & \pm 0.1 \\
\hline $\mathrm{SNh}$ & 25.2 & \pm 0.6 & 18.4 & \pm 0.4 & 14.0 & \pm 0.3 \\
\hline $\mathrm{SNm}$ & 22.4 & \pm 0.5 & 16.0 & \pm 0.3 & 12.3 & \pm 0.2 \\
\hline
\end{tabular}

${ }^{a}$ The effect of the deconvolution with an ideal and a detailed PSF is specified for the model by Steffen (Ch0). The extent of the detailed PSF corresponds to (1) only the size of the model and (2) a periodic repetition of the model to a size of $110^{\prime \prime} \times 110^{\prime \prime}$, which is comparable to the FOV of the observations.

${ }^{b}$ For comparison, the original, i.e., non-degraded contrast values for sets of $\mathrm{CO}^{5} \mathrm{BOLD}$ models (Ch1, Ch2, and $\mathrm{Cm}$ ) and simulations by Stein \& Nordlund (SNh and $\mathrm{SNm}$ ) are given.

pixels with similar heliocentric position $\mu$, effectively forming segments of circles in the images. At disc-centre, the change in $\mu$ over one BFI image is so small that the complete images instead of segments are used. The rms values in Table 3 thus represent the variation among whole images. The smaller the $\mu$ binsize, the smaller the number of the included pixels over which intensity mean and variation are computed. This can lead to a small increase of the scatter in contrast at small $\mu$, while the results for positions closer to the disc-centre are less susceptible. Another way to determine a statistical error is to compare the variation of the contrast in square subfields. This error increases slightly with decreasing subfield size. While the same rms variations than in Table 3 are found for large field sizes, the spread increases slightly to $0.7 \%, 0.6 \%$, and $0.3 \%$ for blue, green, and red continuum, resp., when dividing the images into fields of only $10 " \times 10^{\prime \prime}$.

For the construction of the optimal filter used for deconvolution, assumptions have to be made for the signal model. As already stated in Sect. 2.3, the two different signal models considered here result in contrasts that differ by only $\sim 0.2$ percent points. The deconvolution procedure seems to be rather robust. We degrade the deconvolved images again with the same PSF in exactly the way as it is done for the simulations in Sect. 4. The original observed images and the back-transformed images have contrast values that differ by much less than 0.1 percent points.

\subsection{Synthetic intensity contrast}

For $\mu=1$, the three selected snapshots of the model $\mathrm{Ch} 0$ give an average contrast of $25.0 \%, 18.1 \%$, and $13.8 \%$, at the central 

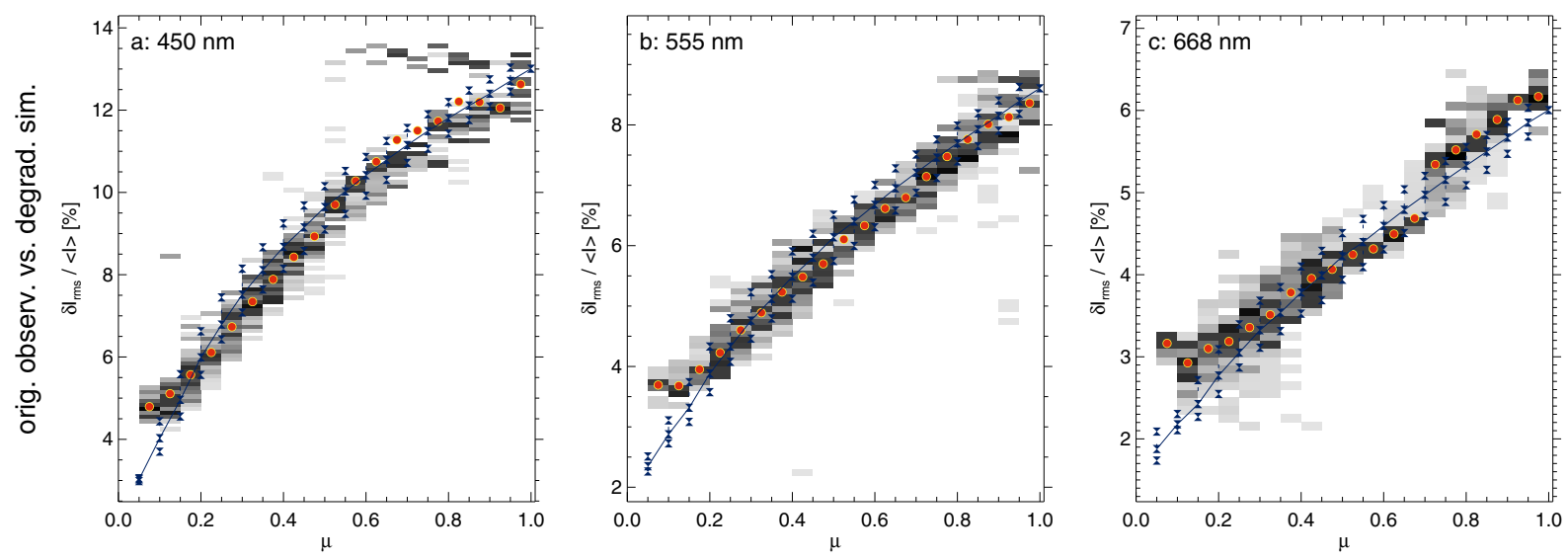

Fig. 7. Centre-to-limb variation of the intensity contrast for the blue (left column), green (middle), and red continuum (right). The contrast values for the SOT wide-band filtergrams are derived from regions of equal $\mu$ (bin size $\Delta \mu=0.05$ ) for all original images. The individual histograms are combined to a set of $\mu$-dependent histograms ("density map"), which are shown as grey scales. The maxima of the histograms are marked with solid circles for each $\mu$ bin. For comparison, the contrast values for the $3 \mathrm{Ch} 0$ snapshots are shown as double triangles, while the solid line represents the mean centre-to-limb variation of model Ch0.

wavelengths of the blue, green, and red continuum channels of the BFI, respectively. The variation among the individual snapshots is for all wavelengths of the order of 0.1 percent point.

Generally, there is a spread of a few tenths of a percent point among individual snapshots. All non-magnetic CO5 BOLD models and also SNh produce very similar contrasts. Obviously, the model Ch0 analysed in more detail in Sect. 4 is no exception but representative for state-of-the-art granulation simulations. Also the weak field model $\mathrm{Cm}$ shows no systematic difference with respect to the non-magnetic models. In contrast, the model with the high field strength (SNm) exhibits systematically lower contrasts with respect to $\mathrm{SNh}$ at disc-centre. The values nevertheless remain roughly of the same order as for the other models but become significantly larger at smaller $\mu$ (see Fig. 8).

The contrast of all non-magnetic and weak field models decreases monotonically with decreasing $\mu$. The difference between the individual simulations can essentially be described as a small contrast offset. An interesting exception is the higher field strength model SNm. Starting with a lower value than SNh at disc-centre, the contrast grows with decreasing $\mu$ until a peak is reached at $\mu=0.3$. For $\mu<0.8, \mathrm{SNm}$ exceeds the contrast of SNh. The blue continuum band of SOT in Fig. 7a indeed shows a secondary group of image segments with a contrast excess, which starts to divert from the main distribution already at disc-centre. It is not obvious at which $\mu$ this subset of SOT observations reaches maximum contrast but it could well be for smaller $\mu$ than seen in the model SNm. The latter represents a plage region with $\langle|B|\rangle=250 \mathrm{G}$, whereas the effect is not clearly discernible for the weak-field model $\mathrm{Cm}$. We therefore argue that the subset of SOT data with contrast excess might contain a small number of magnetic field structures with smaller field strengths.

The deviation of the contrast is connected to the appearance of solar faculae close to the limb. Faculae can be understood as a line-of-sight effect caused by magnetic elements in the intergranular lanes. This topic has been discussed extensively in recent studies by Keller et al. (2004), Carlsson et al. (2004), Steiner (2005), De Pontieu et al. (2006), and Berger et al. (2007) but also by Hirzberger \& Wiehr (2005), Sánchez Cuberes et al. (2003), Sütterlin et al. (1999), and many more. See Ortiz et al. (2002) for an empirical investigation of the centre-to-limb variation of the intensity contrast for solar photospheric faculae.
The additional high-contrast ridge seen in the blue is not visible for the other two SOT channels in Fig. 7. This might partly be a selection effect as we explicitly selected quiet Sun images, avoiding enhanced magnetic field whenever possible. The images were taken during solar minimum so that the Sun was very quiet and not many pronounced active regions were present. Still one has to consider weak magnetic fields on small spatial scales in the quiet Sun.

The small contrast differences among the non-magnetic models are partly systematic effects due to differences in the radiative transfer schemes and in the opacity tables, e.g., PHOENIX/OPAL opacities (Hauschildt et al. 1997; Iglesias et al. 1992) for Ch1. On the other hand, already the random selection of three snapshots influences the mean contrast by the order of a few tenths of a percent point. A larger set of $63 \mathrm{im}-$ ages for $\mathrm{Ch} 2$, for instance, produces contrasts in the blue continuum between $23.7 \%$ and $26.7 \%$ with a mean of $25.2 \%$ and an rms variation of $0.7 \%$. While the mean is reduced by 0.3 percent points for the larger sample, the rms variation increases from $0.5 \%$ to $0.7 \%$ (cf. Table 3 ). A determination of the mean granulation contrast with a precision down to the order of a few $0.1 \%$ obviously requires a statistically significant sample of images rather than a single snapshot.

\section{Spatial power spectral density}

\subsection{Observed power spectral density}

The power spectral density $P_{k}$ (PSD or short "power spectrum") is calculated by integrating the amplitude square $H^{2}$ of the twodimensional Fourier transform of an image over rings of constant wavenumber $k$. We use wavenumbers defined as

$k=\sqrt{k_{x}^{2}+k_{y}^{2}}=2 \pi \sqrt{L_{x}{ }^{-2}+L_{y}{ }^{-2}}$,

where $L_{x}$ and $L_{y}$ are the spatial wavelengths in $x$ - and $y$ direction. Please note that here the one-sided PSD is used, i.e. the Fourier amplitudes $H$ for both the negative and the positive wavenumbers are considered (see, e.g., Press et al. 1992). As the Parseval theorem is fulfilled, the total power for the intensity $I /\langle I\rangle$ is related to the contrast of the original image:

$\int P_{k} \mathrm{~d} k^{\prime}=\left(\delta I_{\mathrm{rms}}\right)^{2}$. 

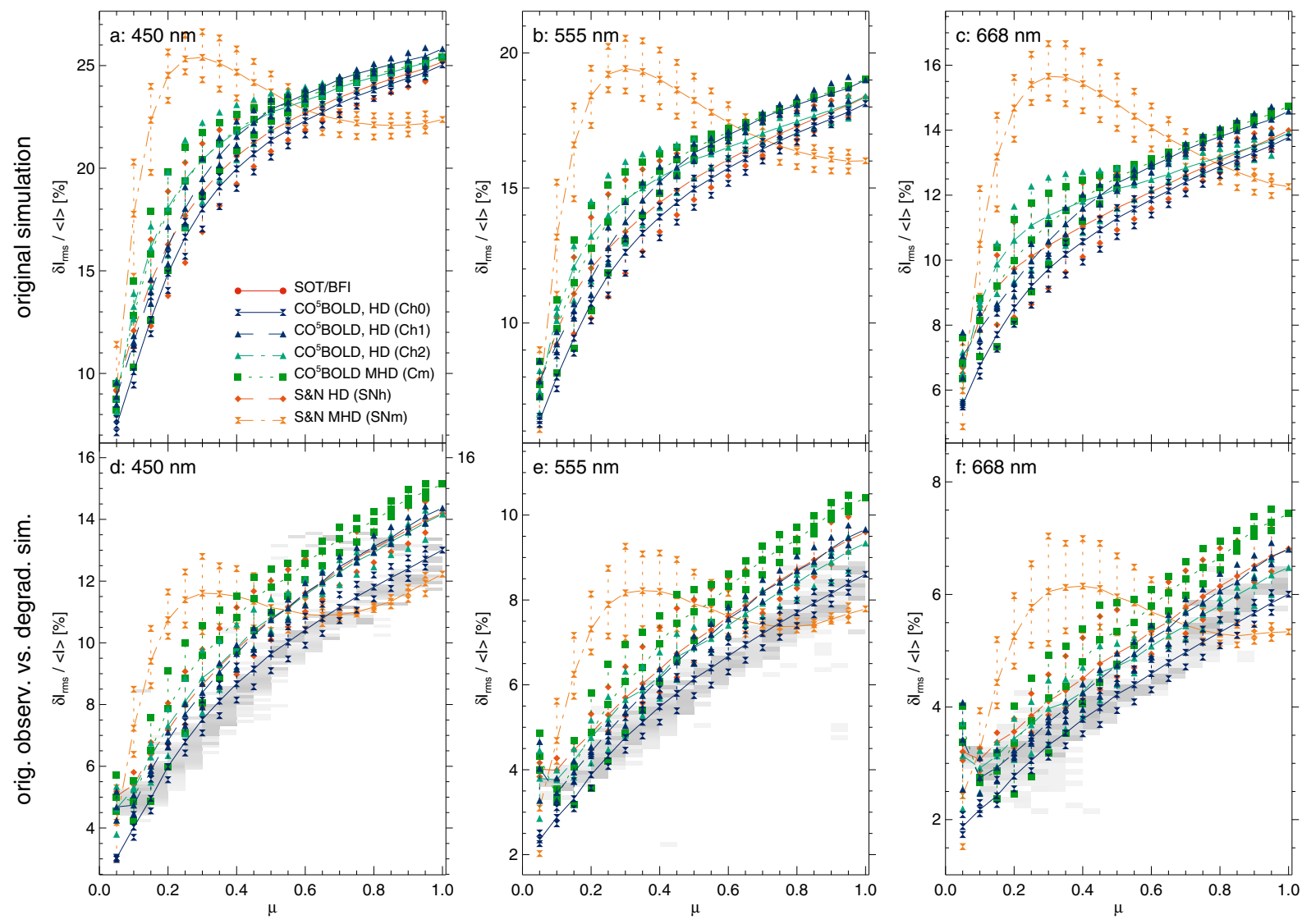

Fig. 8. Centre-to-limb variation of the intensity contrast for all models: top: original synthetic images, bottom: after degradation with the non-ideal PSFs. The columns show the results for the blue (left), green (middle), and red continuum band (right), respectively. The contrast values for the original SOT wide-band filtergrams are represented as gray-shades for reference (cf. Fig. 7) in the lower row. The snapshots of each model are represented by symbols, while the lines show the mean centre-to-limb variation. Please note that only the actual simulation box sizes are considered for the degradation of the synthetic images shown here. Expanding it to a FOV comparable to the SOT observations would lower the individual contrast values but would give practical problems for low $\mu$ (see Sect. 7.3).

Power spectra are calculated for granulation images at disccentre for all three considered SOT continuum bands. The azimuthally integrated power spectra are plotted versus $k$ in the left column of Fig. 9. The curves show power maxima at a spatial scale of $\sim 1.7 \mathrm{Mm}, 1.8 \mathrm{Mm}$, and $1.9 \mathrm{Mm}$ for the blue, green, and red continuum, respectively. That scale is somewhat larger than the most frequent granule diameter, which is typically of the order $\sim 1.4 \mathrm{Mm}$ in the SOT images analysed here. The latter is in very good agreement with earlier results such as, e.g., with the 1".9 reported by Bray \& Loughhead (1977). The spectra also show signs of enhanced power density at spatial scales $>20 \mathrm{Mm}$ that correspond to super-granulation. This is in line with Rieutord et al. (2008), who derive the kinetic energy density spectrum by granule tracking. Except for these additional contributions, the slopes can roughly be approximated with power laws of the form $P_{k} \propto k^{s_{P}}$. The exponents $s_{P}$ for small $k<2.0 \mathrm{Mm}^{-1}$ are of the order of 1.0, i.e. the power increases essentially linearly with wavenumber. Fits of the observational data produce exponents that decrease slightly with wavelength from 1.0 for the blue, to 0.9 for the green, and 0.8 for the red channel. Power law fits are also possible for large wavenumbers although the exponents change with $k$ and thus depend on the considered wavenumber range. In the range from $9 \mathrm{Mm}^{-1}$ to $18 \mathrm{Mm}^{-1}$, we find exponents of roughly $-5.0,-4.5$, and -3.7 for blue, green, and red continuum, respectively. The slopes get steeper at even higher wavenumbers before the power spectral density is dominated by noise and increases again. The contributions to the power spectral density at these wavenumbers are, however, of minor importance only.

The right column of Fig. 9 shows the quantity $k P_{k}$. According to Eq. (3), the area below the curve is equal to the intensity contrast squared. The distributions have clear peaks corresponding to those in the power spectra in the left column but at somewhat smaller spatial scales: $1.4 \mathrm{Mm}$ for blue and red continuum and at $1.5 \mathrm{Mm}$ for the green channel. These scales coincide with the most frequent granule diameter of $\sim 1.4 \mathrm{Mm}$ (see above). The slopes can again be approximated with power laws of the form $k P_{k} \propto k^{S_{P k}}$. The exponents $s_{P k}$ should correspond to $s_{k P}=s_{P}+1$ and are indeed found to be in the range between -4.0 and -2.7 .

\subsection{Synthetic power spectral density}

Not only the intensity distribution but also the power spectral density of model $\mathrm{Ch} 0$ is in line with other simulations as can be seen, e.g., from Fig. 3 by Stein \& Nordlund (2000). It illustrates that model $\mathrm{Ch} 0$ is representative of state-of-art numerical simulations of solar granulation.

\subsection{Comparison of observed and synthetic PSD}

Figure 9 demonstrates that the power spectral density of the degraded synthetic images matches well the observational data described in Sect. 6 for all three continuum bands. The power law 


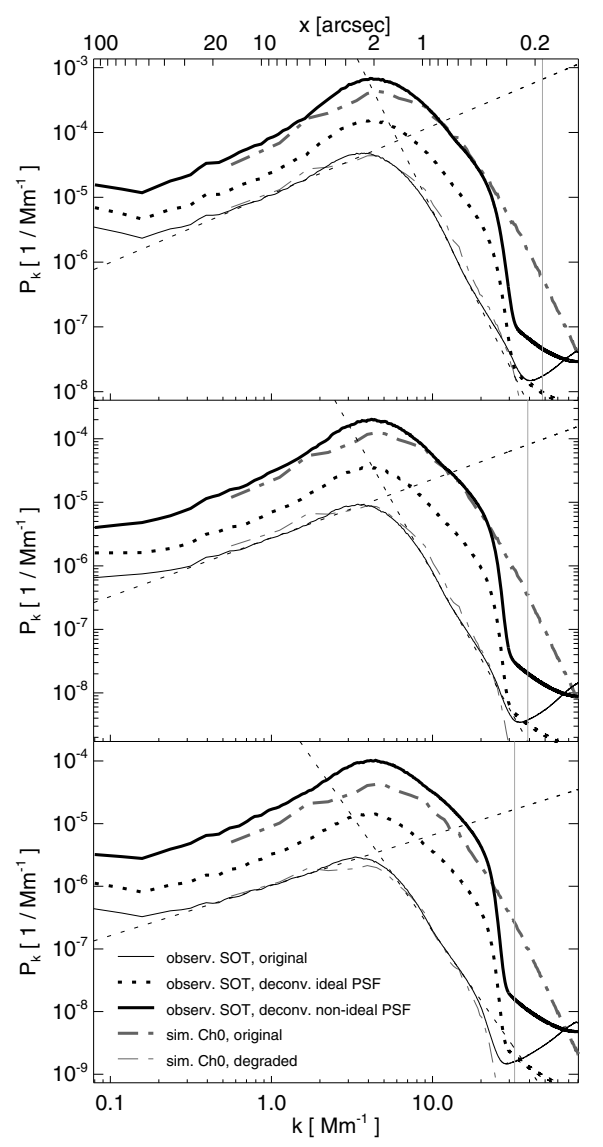

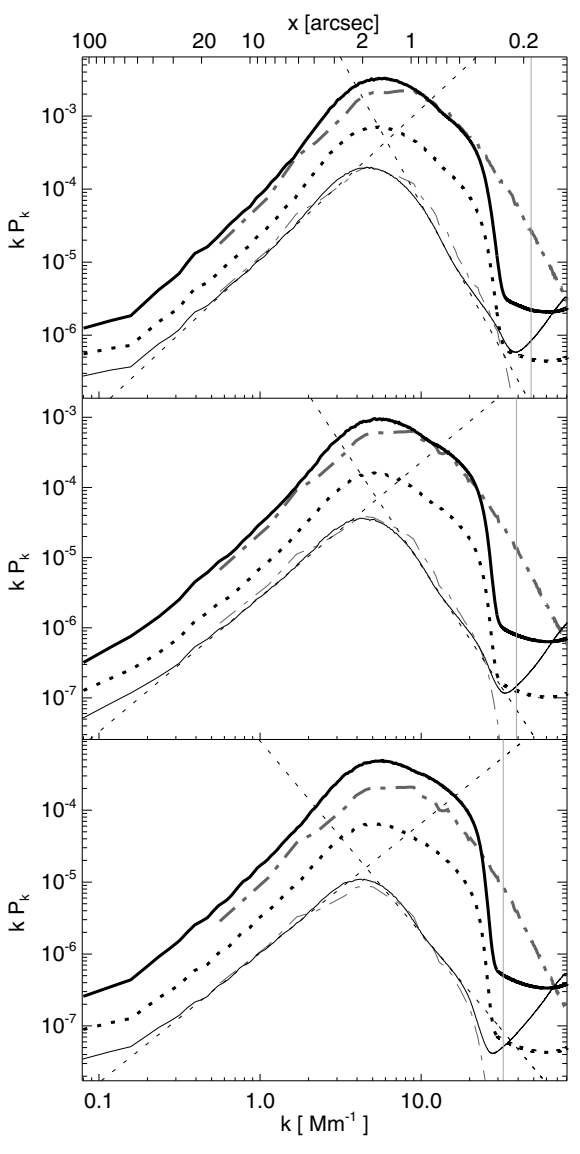

Fig. 9. Power spectral density $P$ (left column) and $k P_{k}$ (right column) for $I /\langle I\rangle$ as functions of spatial wavenumber $k$ for the three BFI continuum wavelengths at disc-centre: blue (top row), green (middle), and red continuum (bottom). The observational data (thin solid) is compared to the the degraded synthetic data (thin dotdashed), while the original synthetic images (thick dot-dashed) are checked against the observations after deconvolution with the ideal PSFs (thick dotted) and the non-ideal PSFs (thick solid). The slopes of the power spectral density can be approximated with powerlaws of the form $P_{k} \propto k^{s_{P}}$. The exponents are $s_{P} \sim 1.0$ for small $k$ and between $\sim-4.0$ and $\sim-5.0$ for large $k$ (dotted lines). For $k P_{k}$, the exponents are correspondingly $s_{k P}=s_{P}+1$ (see text for details). The dotted lines represent the power law fits to the observational data. The solid vertical lines indicate the wavenumber corresponding to the diffraction limit of the telescope. fits for the degraded synthetic data produce exponents that agree very closely with the empirical exponents. The slope for larger wavenumbers in the red continuum, however, is better represented with an exponent of $s_{P} \approx-4.0$, which is slightly smaller than the observational analogue. At these wavenumbers, the instrumental image degradation becomes significant and, by suppressing power at these scales, influences the slopes. The resulting steep decrease in power spectral density is also present for the degraded synthetic images.

The power spectral density for the original synthetic images is generally higher corresponding to a higher intensity contrast (see Eq. (3)). The power law exponents are determined as 1.0 for small wavenumber and $\sim-4.5$ at large wavenumbers for all three wavelengths and thus still agree with the observations. The slight wavelength dependence found for the observed and the degraded data is not present or at least less pronounced in the original synthetic intensity maps. Furthermore, the power spectral density of the latter continues to decrease further with wavenumber well into the domain where the observational signal (and also the degraded synthetic one) is drowned in noise (see Fig. 9), i.e., $k>40 \mathrm{Mm}^{-1}$. Due to that steep slope, the integral of the PSD is changed only slightly when going towards higher wavenumbers. According to the normalisation in Eq. (3), a small change of the integral corresponds to a small change in intensity contrast. The contribution to the intensity contrast is therefore very small at high wavenumbers but large for granulation scales corresponding to smaller $k$.

We illustrate this with an experiment in which we artificially filter away the small scales in synthetic images for the blue band. It is done by Fourier-transforming an image, eliminating all Fourier-components for wavenumbers larger than an imposed cutoff $k_{\text {cutoff }}$, and back-transforming. The power spectral density
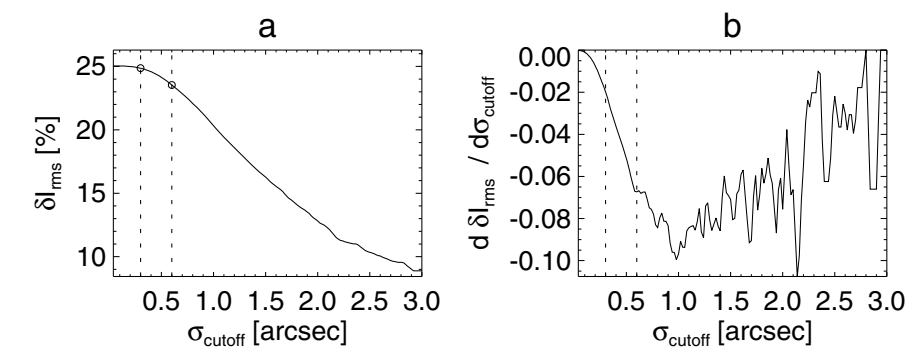

c

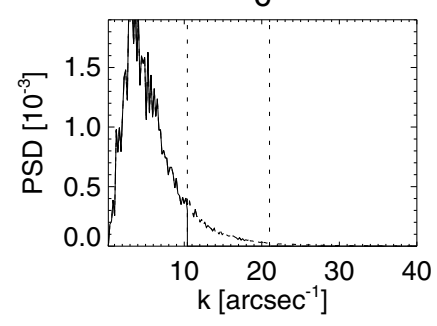

d

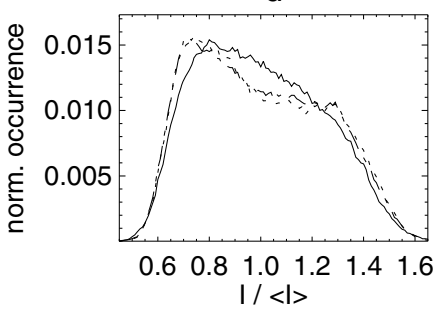

Fig. 10. Fourier-filtering of synthetic images at $450 \mathrm{~nm}$. Intensity contrast $\delta I_{\text {rms }}$ as function of the imposed spatial cutoff $\sigma_{\text {cutoff }}$ a) and its derivative b). The power spectral density c) and the intensity distributions d) are plotted for the original images (dotted lines) and two selected cutoffs of 0.3 (dot-dashed) and $0 . .6$ (solid), which are marked as open circles and/or vertical dotted lines in panels a)-c).

of the resulting image is thus truncated at $k_{\text {cutoff }}$ (see Fig. 10c). The intensity contrast of the filtered images is plotted in Fig. 10a as function of the spatial cutoff scale $\sigma_{\text {cutoff }}=2 \pi / k_{\text {cutoff }}$. The contrast decreases monotonically with the cutoff. Towards the smallest scales, $\delta I_{\text {rms }}$ saturates to the value of unfiltered images $(25.0 \%)$. The asymptotic approach to the final value already 
starts at scales that are well resolved in the numerical model (grid size $\sim 0$ ".039). The change of the slope can be seen from derivative $\delta I_{\text {rms }} / \sigma_{\text {cutoff }}$ in Fig. 10b. (The derivative at larger $\sigma_{\text {cutoff }}$ is noisy due to the numerical treatment of the discrete Fourier filtering.) The biggest change of the contrast occurs at scales between $>0$ ". 5 and $\sim 2$ ". 2 , which coincides with the "granulation peak" in the PSD. In contrast, the change towards the smallest scales decreases quickly. Removing the smallest scales has apparently only a small effect in terms of contrast. A cutoff of $\sigma_{\text {cutoff }}=0$. .5 still produces a contrast of $24.2 \%$, i.e., a reduction of only 0.8 percent points. The absence of small scales is more obvious when looking at the intensity histograms in Fig. 10d. The distributions for the unfiltered images and for $\sigma_{\text {cutoff }}=0$ ". 3 are very similar, whereas $\sigma_{\text {cutoff }}=0$ ".6 already produces noticeable differences. The otherwise clearly visible double-peak transforms into a single-peaked asymmetric distribution. The reduced occurrence of the brightest and darkest pixels implies that they are connected to the smallest scales. The darkest pixels, which constitute the "major component of the intensity distribution (at $I<\langle I\rangle$, cf. Sect. 4.1 and Figs. 6 and 3), are found in the narrow intergranular lanes. In the case of SOT, the lanes seem to be sufficiently resolved. We therefore expect no change of the contrast numbers for deconvolved images from the use of telescopes with larger apertures. See, e.g., Rao et al. (2001) for an analysis of the granulation contrast as function of the telescope aperture.

Finally, we compare the PSD derived from the deconvolved observations with those for the original synthetic images, which are all plotted as thick lines in Fig. 9. The dotted and solid lines represent the PSD for deconvolution with the ideal and the nonideal PSFs, respectively. The original simulations are marked with the dot-dashed lines. While deconvolution with the ideal PSF already produces a significant increase in power througout the observationally accessible wavenumber range, the non-ideal PSFs bring the observations very close to the simulation results. The PSDs agree well for the blue and green continuum. For the red continuum, however, we remain with a small discrepancy, which is consistent with the differences in terms of intensity contrast (see Table 3). The sharp decrease of the deconvolved PSDs close to the theoretical cutoff wavenumber (vertical lines in Fig. 10) results in a significant power deficit with respect to the simulations. This is simply due to the fact that there is no further information content in the images at the corresponding spatial scales due to the limited spatial resolution. Consequently, the power contributions at wavenumber close to the spatial cutoff are lost.

\section{Discussion}

\subsection{Summary}

Both simulations and observations are found to have a considerable variation in contrast. It is mostly a statistical variation between different snapshots/images. And still there are systematic, physical sources to be considered. Title et al. (1989; see also Spruit et al. 1990) have shown that removing the intensity modulation due to f- and p-mode oscillations in the data taken with SOUP at the former Swedish Vacuum Solar Telescope reduces the rms intensity variations by $35 \%$. The relative contribution of oscillations to the contrast depends on the spatial resolution as oscillations are correlated with certain spatial scales of which some possibly cannot be resolved (see, e.g., Spruit et al. 1990).
We conclude that in view of the mutual variation ranges one cannot expect a much better match of synthetic and observational continuum intensity contrast than has been achieved here.

\subsection{Comparison with other studies}

Observed contrast values for quiet Sun granulation vary significantly throughout the literature, indicating the large uncertainties arising from often unknown properties of the employed instrument and seeing effects. See Sánchez Cuberes et al. (2000) for an extensive survey of observed contrast values. Here we discuss some of the more recent studies that report granulation contrast measurements.

Khomenko et al. (2003) determined a granulation contrast between 5 and $6 \%$ for the continuum around $500 \mathrm{~nm}$ from observations with the VTT. Our SOT data rather indicate a contrast value of $\sim 11 \%$ for flat-fielded images at this wavelength. Note that Khomenko et al. (2003) make no corrections for instrumental and seeing effects.

Also using the VTT, de Boer (1995) found a contrast of $14.5 \%$ (cf. Pérez Rodríguez \& Kneer 2002) in speckle reconstructed images at $550 \mathrm{~nm}$, which is significantly lower than the $19.4 \%$ we derived for the green continuum channel of SOT/BFI after deconvolution with the non-ideal PSF.

Van Noort et al. (2005) report granulation contrast numbers for SST observations in the continuum at $436.4 \mathrm{~nm}$. Their images are restored with the MOMFBD image restoration technique and have maximum contrast values slightly below $11 \%$. These values are significantly lower than the $12.8 \%$ that we find for the SOT/BFI blue continuum at slightly longer wavelength before deconvolution (see Table 3). The PSFs they derived from MOMFBD processing are based on sub-images of size $\sim 5^{\prime \prime} \times 5^{\prime \prime}$ and can therefore not account for the far wings of the PSF. No correction for scattered light is applied through MOMFBD processing. As we will discuss later in this section, this is probably the main reason why their contrast values are relatively low.

Uitenbroek et al. (2007) compare speckle-reconstructed G-band images of the quiet Sun taken with the Dunn Solar Telescope (DST) with a synthetic image calculated from a snapshot of radiation magnetohydrodynamical simulation by Stein \& Nordlund (2006) with an initially horizontal magnetic field with $\left\langle\left|B_{0}\right|\right\rangle=30 \mathrm{G}$. They find a considerable discrepancy between the observed contrast of $14.1 \%$ for their best reconstructed images, and the synthetic contrast of $21.5 \%$. Uitenbroek et al. also analyse a synthetic image from the same Stein \& Nordlund simulation with $\left\langle B_{z}\right\rangle=250 \mathrm{G}$ that we use here (see Sect. 3.1). They determine that the contrast of this snapshot, which corresponds to a plage region, is only $16.3 \%$ in the G-band. The fact that the effect of increasing the magnetic field leads to a decrease in the contrast, leads them to speculate that the quiet Sun might contain a larger amount of weak magnetic field than the $30 \mathrm{G}$ assumed for the quiet Sun model.

Our comparison of synthetic contrast values in the continuum around $\lambda=450 \mathrm{~nm}$ in Sect. 5.3 also shows that a higher average magnetic field strength lowers the contrast (see Table 3) but to much smaller extent than found by Uitenbroek et al. in the G-band. This suggests that the presence of a large amount of spectral lines in the G-band is an important factor for the value of the granulation contrast as one compares magnetically active and quiet regions.

We analyse speckle-reconstructed images obtained with the Dutch Open Telescope (DOT, Rutten et al. 2004), which we obtained from the DOT online data base. We find contrast values 
ranging between $14.4 \%$ to $18.6 \%$ for the G-band and values in the range from $14.9 \%$ to $19.8 \%$ for the DOT blue continuum at $432 \mathrm{~nm}$. The code used to reconstruct the DOT images is based on an IDL code first mentioned in de Boer \& Kneer (1992). While some of the DOT contrast values are close to the DST result from Uitenbroek et al. (2007), others are clearly higher. Uitenbroek et al. find values around $14 \%$ for G-Band images obtained with the DST using the speckle code described in detail in Wöger \& von der Lühe (2008). This code is different from the code used for the aforementioned DOT observations. The code by Wöger \& von der Lühe takes into account the usage of an Adaptice Optics (AO) system. The functions used for the Fourier amplitude calibration are modified for Adaptive Optics usage according to Wöger \& von der Lühe (2007). See Wöger et al. (2008) for an analysis of the code's photometric accuracy in comparison to the Hinode satellite.

The previous paragraphs illustrate that there is a considerable spread among the contrast values of reconstructed images due to inherent sources of uncertainty. In the case of speckle interferometry, uncertainties in the Fourier amplitude calibration due to an incorrect estimation of the Fried parameter and/or (if applicable) the AO correction performance - and thus the application of the wrong calibration function - can lead to a large scatter in contrasts for the reconstructed images.

In general, instrumental straylight is not accounted for in neither the speckle nor the MOMFBD reconstruction process, and needs to be treated separately. Therefore, the contrast values from post-facto reconstructed images should at best be compared to those disc-centre images of SOT that have been deconvolved with the ideal PSF only (e.g., $(18.0 \pm 0.8) \%$ for the blue continuum). But even then it is not guaranteed that the effect of seeing is completely removed while this source of uncertainty is of no concern for the space-bourne measurements with Hinode.

The apparent contrast dilemma can be solved by reviewing the problematic treatment of non-ideal PSF contributions. The determination of the straylight level is very difficult, in particular for ground-based observations. Consequently, details of the straylight properties are often unknown. A frequent strategy to account for straylight is to simply assume a constant contribution $\alpha$, which is equally spread over the FOV. It corresponds to an intensity offset, which decreases the intensity contrast to $(1-\alpha)$ of its original value. Uitenbroek et al. (2007) conclude that they would need a straylight level of $\alpha=0.34$ in order to reproduce the contrast in their synthetic images. They consider this a factor 3 too high for the DST. We also determine $\alpha$ from the difference between SOT images deconvolved with just the ideal diffraction-limited PSF and the full non-ideal PSF (see Table 3). In case of the blue continuum at disc-centre, the contrast difference of 8.5 percent points translates into $\alpha=0.33$. For the green and red continuum, we find 0.37 and 0.41 , respectively. Although these values are very close to the one by Uitenbroek et al., they should not be interpreted as actual straylight levels. The eclipse and Mercury transit observations, on which the SOT PSF with non-ideal Voigt-function contributions is based on, show residual intensities of the order of a few percent points only (see Paper I). Obviously, the assumption of a constant straylight contribution $\alpha$ is misleading. A constant offset would not only influence the PSF wings as intended but also the PSF core. For SOT, the straylight level was actually shown to be dependent on the mean count rate, i.e., the overall light level in the instrument, and with that on the heliocentric position of the FOV. We therefore argue that a more detailed straylight model is crucial for detailed comparions of the granulation contrast and the intensity distributions in general.
Danilovic et al. (2008) obtained a continuum intensity map at $630 \mathrm{~nm}$ wavelength with the spectro-polarimeter (SP) of SOT. For this single map, they report a contrast of $7.0 \%$, a value which is in line with our results from SOT/BFI. After detailed modelling of the SOT/SP optical system, they degrade a synthetic image from a MHD simulation by Vögler \& Schüssler (2007) taking into account a detailed PSF. For the degraded synthetic image, they find a contrast of 8.5\%. Danilovic et al. (2008) argue that a slight defocus of the SP instrument can account for a further decrease of the synthetic contrast of $1 \%$. The remaining $0.5 \%$ discrepancy is then attributed to straylight and imperfections in the optical system, although one could also argue for an intrinsic spread in the observed contrast values. Our results point at a range of observed contrast values on the order of a few tenths of a percent around $630 \mathrm{~nm}$.

In their recent paper, Trujillo Bueno \& Shchukina (2009) present the rms granulation contrast derived from detailed 3D radiative transfer for the $3 \mathrm{D}$ hydrodynamic model by Asplund et al. (2000). Judging from their Fig. 9, the synthetic intensity maps have contrasts of roughly $24 \%, 17 \%$, and $12 \%$ for wavelengths corresponding to the blue, green and red channels of the BFI. These values are systematically lower than for the synthetic maps considered here (see Table 3). The difference, however, is well within the variation range of individual observed images and uncertainties discussed in Sect. 7.3.

\subsection{Additional sources of uncertainty}

Spectrum synthesis. In Linfor3D, pure local thermodynamic equilibrium is assumed, i.e. scattering is not taken into account. In their Fig. 8, Trujillo Bueno \& Shchukina (2009) present the centre-to-limb variation of the continuum intensity at $\lambda=$ $300 \mathrm{~nm}$. They demonstrate that accounting for the scattering contribution to the source function removes the discrepancy between the observed and synthetic intensity. The discrepancy, however, is only of the order of a few percent points for small $\mu$ and vanishes towards disc-centre. Scattering is even less important for the wavelengths considered here. We therefore argue that the neglect of scattering in Linfor3D has only small if not negligible influence on our results.

Point spread function. As the conditions may vary somewhat between different observations, the corresponding PSF also varies. Consequently, each image would require its very own specific PSF for deconvolution. In practice, it was only possible to determine PSFs for BFI/SOT that represent the best-fit cases for a large range in observational conditions (see Paper I). They may be considered as representative of a typical observational situation. The analysis of the deconvolved images should therefore be based on a statistically significant set of images.

Also the degradation of the synthetic images with the PSF bears sources of uncertainty. The spatial dimensions of the simulations used here are smaller than for the observed images and the extent of the PSFs from Paper I. The convolution of a synthetic map with a PSF therefore mainly takes into account the central parts of the PSF while the far wings are effectively neglected. As mentioned already in Sect. 4.2, one should consider a FOV that is comparable to the one of the observation. Periodical extension of the synthetic maps to a size of $110^{\prime \prime} \times 110^{\prime \prime}$ and degradation by a correspondingly larger PSF, lead to a reduced intensity contrast for the model by Steffen (see Table 3). A lower contrast of the synthetic maps seems actually to be in line with 
the difference between the contrasts of the deconvolved observations and the original synthetic maps.

The influence of a too small FOV can also be seen for the other models in Fig. 8. Except for the model with higher magnetic field strength, all simulations produce contrast values for the undegraded images that lie within their mutual variation ranges (see right column in the figure). After degradation (middle column), however, a systematic shift can be seen. The $\mathrm{CO}^{5} \mathrm{BOLD}$ models are stacked according to the size of their spatial extent: low contrasts for model by Steffen to higher contrasts for the smallest model $\mathrm{Cm}$. The systematic difference can be removed by consistently going towards a large FOV of the same size. Making a realistic extension of the FOV is only practically feasible for disc-centre images. For regions towards the limb, however, the extension of the FOV would require to vary the observing angle across the image. As there is no straightforward procedure to do this, we refrain from enlarging the PSF for the calculation of the centre-to-limb variation of the contrast.

The uncertainty in the PSF wing contribution (as seen from the choice of the FOV) is counteracted by uncertainties in the PSF parameters. In the following, we illustrate the effect for the blue continuum images. So far we used the best-fit PSF as determined in Paper I. The PSF for the blue BFI channel is determined by the Voigt parameters $\gamma=0$ ".004 and $\sigma=0$ ". 008 (see Sect. 2.2). Application of this PSF produces mean contrasts of $13.0 \%$ and $11.3 \%$ for the small and the large FOV, respectively. We now consider the error margins for the non-ideal Voigt contribution to the PSF as given in Table 4 in Paper I. First we remain with $\gamma=0$ ".004 and shift $\sigma$ according to the uncertainties of the linear regression stated in Table 4 in Paper I. The resulting mean contrast range from $12.9 \%$ to $13.4 \%$ for the small FOV to $11.2 \%$ and $11.8 \%$ for the large FOV. The spread in possible contrast values becomes larger if also the uncertainty in the parameter $\gamma$ is considered. Within the specified error ranges, we can produce contrasts up to $13.2 \%$ or even $13.6 \%$. The intrinsic uncertainty in the PSF apparently allows for the contrasts of the degraded synthetic maps in the range of $\sim 11.2 \%$ to $\sim 13.6 \%$. The observational result of $12.8 \%$ is well included in this range. In return, the uncertainty in the non-ideal PSF contribution would also lead to corresponding error margins for the deconvolved images so that also the original synthetic images and the deconvolved observations agree in terms of contrast within their mutual error margins.

Limb observations. The solar limb is used to re-determine the coordinates of the FOV whenever it can be seen in an image. The derived positions sometimes differ from the Hinode coordinates in the image file headers by of the order of $1^{\prime \prime}$ or less. At the limb, a small pointing error can translate into a significant uncertainty in $\mu$ (e.g.: $\Delta \mu \approx 0.05$ for $1^{\prime \prime}$ ). The unavoidable smearing due to the limited spatial resolution of an instrument limits the accuracy of the limb determination. The uncertainty can be given as $\Delta \mu<0.03$ for the BFI continuum bands, when assuming one FWHM of the PSF core as maximum offset. Effects like thermal deformation of the instrument structure and fluctuations in the flight characteristics of the spacecraft induce an additional jittering. Kosugi et al. (2007) state that the image stabilization system of SOT achieves 0 ".007 ( $3 \sigma$ level) and effectively restricts the rms image displacement to less than 0 ".03 (Tsuneta \& et al. 2007; Kosugi et al. 2007). The influence of the spacecraft on the spatial resolution should thus be rather insignificant. We nevertheless explicitly state that data for $\mu<0.1$, corresponding to distances less than $\sim 5^{\prime \prime}$ from the limb, has to be regarded with great care in view of the potential error margins.

Power excess and JPEG compression. In Sect. 2.3, we mentioned a small power contribution at spatial scales between the granulation peak and the spatial resolution of SOT (see Fig. 9). Wöger et al. (2008) describe a similar power excess between SOT and DST data (see their Fig. 5). It is clearly in excess compared to synthetic images and also is usually not seen in comparable SST data. We therefore argue that the power excess is an artefact. As the enhanced power peak occurs at frequencies that correspond to roughly 8 pixels and less (see Fig. 9), it could in principle be related to JPEG compression. Essentially all SOT images are compressed before downlinking to the ground in order to increase the telemetry throughput. The used lossy JPEG image compression uses a $8 \times 8$ pixel blocking. Consequently artefacts can be expected on pixel scales of 8 and harmonics thereof. We therefore compressed and decompressed synthetic images with routines kindly provided by Katsukawa \& Tsuneta (priv. comm.). The power spectral density of the resulting processed images do not significantly differ from those of the original images, disqualifying the JPEG compression as the source. Whatever the cause of the artefact, the effect on the intensity contrast is negligible as the affected scales do not contribute much to the overall contrast.

\section{Conclusion}

State-of-the-art radiation (magneto-)hydrodynamic simulations can reproduce many properties of the observed continuum intensity distribution if the effects of instrumental image degradation are properly taken into account. The latter can have dramatic effects. Although SOT is an excellent instrument, which works close to the diffraction limit, the non-ideal component to the PSF has a significant effect on the intensity distribution. The removal of the degradation by PSF-deconvolution more than doubles the intensity contrast. In contrast to earlier studies, we derive final contrast values at blue, green, and red continuum wavelengths that are slightly higher than for the original synthetic images, although they still agree within the mutual variation ranges. A detailed realistic treatment of instrumental straylight is found to be of crucial importance for a quantitative comparison between simulations and observations. A constant straylight contribution alone is not sufficient and misleading.

Owing to the many sources of uncertainty, the intensity contrast as a single number is a poor mean of comparing observations and simulations. This is already problematic for comparisons of observations that are taken with different instruments or under different conditions. The only practicable way is to properly remove the effects of image degradation prior to comparison as it is demonstrated here for SOT. This procedure is unfortunatly not always possible as the properties of the PSF are often poorly known. However, even in such rather fortunate cases as for SOT, one cannot expect an exact match between observations and simulations. Already the uncertainties in the non-ideal PSF contributions, result in a error margins of the order of 1 or possibly even 2 percent points in the blue continuum.

Comparisons should therefore be based on more informative properties of the intensity distribution. Here we find the intensity histograms and the power spectral density from observation and simulation to agree very well. It is obvious that all spatial scales with significant contributions to the contrast are resolved with the $50-\mathrm{cm}$ aperture of SOT. Therefore we do not expect that 
observations with larger aperture telescopes would yield different contrast values for the quiet Sun. We finally conclude that the traditionally perceived conflict between observations and simulations can now be dismissed.

Acknowledgements. The authors thank M. Carlsson, M. Steffen, F. Wöger, H.-G. Ludwig, O. Steiner, Ø. Langangen, S. Tsuneta, and O. von der Lühe for helpful discussions. S. Haugan and T. Fredvik are acknowledged for their support with the Hinode data centre. This work was supported by the Research Council of Norway, grant 170935/V30, and a Marie Curie Intra-European Fellowship of the European Commission (6th Framework Programme, FP62005-Mobility-5, Proposal No. 042049). Intensive use was made of the Hinode Science Data Centre Europe hosted by the Institute of Theoretical Astrophysics of the University of Oslo, Norway. Hinode is a Japanese mission developed and launched by ISAS/JAXA, collaborating with NAOJ as a domestic partner, NASA and STFC (UK) as international partners. Scientific operation of the Hinode mission is conducted by the Hinode science team organized at ISAS/JAXA. This team mainly consists of scientists from institutes in the partner countries. Support for the post-launch operation is provided by JAXA and NAOJ (Japan), STFC (U.K.), NASA, ESA, and NSC (Norway). This research has made use of NASA's Astrophysics Data System.

\section{References}

Asplund, M., Nordlund, Å., Trampedach, R., Allende Prieto, C., \& Stein, R. F. 2000, A\&A, 359, 729

Berger, T. E., van der Voort, L. R., \& Löfdahl, M. 2007, ApJ, 661, 1272

Bray, R. J., \& Loughhead, R. E. 1977, Sol. Phys., 54, 319

Carlsson, M., Stein, R. F., Nordlund, Å., \& Scharmer, G. B. 2004, ApJ, 610, L137

Danilovic, S., Gandorfer, A., Lagg, A., et al. 2008, A\&A, 484, L17

de Boer, C. R. 1993, Ph.D. Thesis, Göttingen

de Boer, C. R. 1995, A\&AS, 114, 387

de Boer, C. R., \& Kneer, F. 1992, A\&A, 264, L24

De Pontieu, B., Carlsson, M., Stein, R., et al. 2006, ApJ, 646, 1405

Deubner, F. L., \& Mattig, W. 1975, A\&A, 45, 167

Freeland, S., Bentley, R., \& Murdin, P. 2000, Encyclopedia of Astronomy and Astrophysics

Freytag, B., Steffen, M., \& Dorch, B. 2002, Astron. Nachr., 323, 213

Freytag, B., Steffen, M., Ludwig, H.-G., \& Wedemeyer-Boehm, S. 2008, Astrophysics Software Database, 36

Gonzalez, R., \& Woods, R. 2008, Digital Image Processing (Pearson Prentice Hall)

Gustafsson, B., Edvardsson, B., Eriksson, K., et al. 2008, A\&A, 486, 951

Hauschildt, P. H., Baron, E., \& Allard, F. 1997, ApJ, 483, 390

Hirzberger, J., \& Wiehr, E. 2005, A\&A, 438, 1059

Ichimoto, K., Katsukawa, Y., Tarbell, T., et al. 2008, in First Results From Hinode, ed. S. A. Matthews, J. M. Davis, \& L. K. Harra, ASP Conf. Ser., 397,5

Iglesias, C. A., Rogers, F. J., \& Wilson, B. G. 1992, ApJ, 397, 717

Keller, C. U. 2006, in Solar MHD Theory and Observations: A High Spatial Resolution Perspective, ASP Conf. Ser., ed. J. Leibacher, R. F. Stein, \& H. Uitenbroek, 3, 354

Keller, C. U., Schüssler, M., Vögler, A., \& Zakharov, V. 2004, ApJ, 607, L59

Khomenko, E. V., Collados, M., Solanki, S. K., Lagg, A., \& Trujillo Bueno, J. 2003, A\&A, 408, 1115

Khomenko, E. V., Martínez González, M. J., Collados, M., et al. 2005, A\&A, 436, L27

Kiselman, D. 2008, Physica Scripta Volume T, 133, 014016
Kosugi, T., Matsuzaki, K., Sakao, T., et al. 2007, Sol. Phys., 243, 3

Kupka, F., Piskunov, N., Ryabchikova, T. A., Stempels, H. C., \& Weiss, W. W. 1999, A\&AS, 138, 119

Langangen, Ø., Carlsson, M., Rouppe van der Voort, L., \& Stein, R. F. 2007, ApJ, 655, 615

Langhans, K., \& Schmidt, W. 2002, A\&A, 382, 312

Leenaarts, J., Rutten, R. J., Sütterlin, P., Carlsson, M., \& Uitenbroek, H. 2006, A\&A, 449, 1209

Mattig, W. 1983, Sol. Phys., 87, 187

Muller, R., Hanslmeier, A., \& Saldaña-Muñoz, M. 2007, Mem. Soc. Astron. It., 78,71

Neckel, H., \& Labs, D. 1984, Sol. Phys., 90, 205

Nordlund, A. 1984, in Small-Scale Dynamical Processes in Quiet Stellar Atmospheres, ed. S. L. Keil, 174

Ortiz, A., Solanki, S. K., Domingo, V., Fligge, M., \& Sanahuja, B. 2002, A\&A, 388,1036

Pérez Rodríguez, E., \& Kneer, F. 2002, A\&A, 395, 279

Press, W. H., Teukolsky, S. A., Vetterling, W. T., \& Flannery, B. P. 1992, Numerical recipes in FORTRAN. The art of scientific computing, 2nd edn. (Cambridge: University Press)

Rao, C.-H., Jiang, W.-H., Ling, N., \& Beckers, J. M. 2001, Chinese Astronomy and Astrophysics, 25, 439

Rieutord, M., Meunier, N., Roudier, T., et al. 2008, A\&A, 479, L17

Rouppe van der Voort, L. H. M., Löfdahl, M. G., Kiselman, D., \& Scharmer, G. B. 2004, A\&A, 414, 717

Rutten, R. J., Hammerschlag, R. H., Bettonvil, F. C. M., Sütterlin, P., \& de Wijn, A. G. 2004, A\&A, 413, 1183

Sánchez Cuberes, M., Bonet, J. A., Vázquez, M., \& Wittmann, A. D. 2000, ApJ, 538,940

Sánchez Cuberes, M., Vázquez, M., Bonet, J. A., \& Sobotka, M. 2003, A\&A, 397, 1075

Schaffenberger, W., Wedemeyer-Böhm, S., Steiner, O., \& Freytag, B. 2006, in Solar MHD Theory and Observations: A High Spatial Resolution Perspective, ed. J. Leibacher, R. F. Stein, \& H. Uitenbroek, ASP Conf. Ser., 354, 345

Shimizu, T., Nagata, S., Tsuneta, S., et al. 2008, Sol. Phys., 249, 221

Spruit, H. C., Nordlund, A., \& Title, A. M. 1990, ARA\&A, 28, 263

Steffen, M. 2007, in IAU Symposium, ed. F. Kupka, I. Roxburgh, \& K. Chan, IAU Symp., 239, 36

Stein, R. F., \& Nordlund, Å. 1998, ApJ, 499, 914

Stein, R. F., \& Nordlund, Å. 2000, Sol. Phys., 192, 91

Stein, R. F., \& Nordlund, À. 2006, ApJ, 642, 1246

Steiner, O. 2005, A\&A, 430, 691

Suematsu, Y., Tsuneta, S., Ichimoto, K., et al. 2008, Sol. Phys., 249, 197

Sütterlin, P., Wiehr, E., \& Stellmacher, G. 1999, Sol. Phys., 189, 57

Title, A. M., Tarbell, T. D., Topka, K. P., et al. 1989, ApJ, 336, 475

Trujillo Bueno, J., \& Shchukina, N. 2009, ApJ, 694, 1364

Tsuneta et al. 2007, ArXiv e-prints, 711

Tsuneta, S., Ichimoto, K., Katsukawa, Y., et al. 2008, Sol. Phys., 249, 167

Uitenbroek, H., Tritschler, A., \& Rimmele, T. 2007, ApJ, 668, 586

van Noort, M., Rouppe van der Voort, L., \& Löfdahl, M. G. 2005, Sol. Phys., 228,191

Vögler, A., \& Schüssler, M. 2007, A\&A, 465, L43

von der Luehe, O. 1993, A\&A, 268, 374

Wedemeyer, S., Freytag, B., Steffen, M., Ludwig, H.-G., \& Holweger, H. 2004, A\&A, 414, 1121

Wedemeyer-Böhm, S. 2008, A\&A, 487, 399

Wilken, V., de Boer, C. R., Denker, C., \& Kneer, F. 1997, A\&A, 325, 819

Wöger, F., \& von der Lühe, O. 2007, Appl. Opt., 46, 8015

Wöger, F., \& von der Lühe, O. 2008, in Society of Photo-Optical Instrumentation Engineers (SPIE) Conf. Ser., 7019

Wöger, F., von der Lühe, O., \& Reardon, K. 2008, A\&A, 488, 375 